ARTICLE

\title{
Structural correlates of affinity in fetal versus adult endplate nicotinic receptors
}

Tapan Kumar Nayak ${ }^{1, \star}$, Srirupa Chakraborty ${ }^{1, \star}$, Wenjun Zheng $^{2} \&$ Anthony Auerbach $^{1}$

Adult-type nicotinic acetylcholine receptors (AChRs) mediate signalling at mature neuromuscular junctions and fetal-type AChRs are necessary for proper synapse development. Each AChR has two neurotransmitter binding sites located at the interface of a principal and a complementary subunit. Although all agonist binding sites have the same core of five aromatic amino acids, the fetal site has $\sim 30$-fold higher affinity for the neurotransmitter ACh. Here we use molecular dynamics simulations of adult versus fetal homology models to identify complementary-subunit residues near the core that influence affinity, and use singlechannel electrophysiology to corroborate the results. Four residues in combination determine adult versus fetal affinity. Simulations suggest that at lower-affinity sites, one of these unsettles the core directly and the others (in loop E) increase backbone flexibility to unlock a key, complementary tryptophan from the core. Swapping only four amino acids is necessary and sufficient to exchange function between adult and fetal AChRs.

\footnotetext{
${ }^{1}$ Department of Physiology and Biophysics, State University of New York at Buffalo, Buffalo, New York 14214, USA. ${ }^{2}$ Department of Physics, State University of New York at Buffalo, Buffalo, New York 14260, USA. * These authors contributed equally to this work. Correspondence and requests for materials should be addressed to A.A. (email: auerbach@buffalo.edu).
} 
ndplate AChRs are heteropentamers that have two $\alpha(1)$ - subunits and one each of $\beta, \delta$ and either $\gamma$ or $\varepsilon$. Each receptor has two functional neurotransmitter binding sites located in the extracellular domain at subunit interfaces, either $\alpha \delta+\alpha \gamma$ (fetal) or $\alpha \delta+\alpha \varepsilon$ (adult) (Fig. 1a, inset). Agonist affinities in mouse AChRs have been measured for these sites, both separately and as pairs ${ }^{1-4}$. The resting equilibrium dissociation constant for ACh $\left(K_{\mathrm{d}}^{\mathrm{ACh}}\right)$ is $\sim 30$-fold lower at $\alpha \gamma$ compared with $\alpha \delta$ or $\alpha \varepsilon$. The affinity of the fetal, $\alpha \gamma$-site is similar to that of the Lymnaea stagnalis acetylcholine binding protein $(\mathrm{AChBP})^{5}$.

The fetal, $\gamma$-subunit is required for the proper maturation of the neuromuscular synapse ${ }^{6-8}$. In mice, $\gamma$-null mutations are lethal ${ }^{9}$ and in humans $\gamma$-subunit mutations cause both lethal and non-lethal (Escobar) types of multiple pterygium syndromes ${ }^{10-12}$. The reason(s) for the $\gamma$-subunit requirement in synaptogenesis is not known, but possibilities include the higher agonist affinity, smaller single-channel conductance, longer open-channel lifetime, smaller gating-voltage dependence, lower $\mathrm{Ca}^{++}$ permeability and lower probability of opening constitutively of fetal AChRs 1,13,14. Physiologically, the higher affinity of the fetal agonist binding site for both ACh and choline will lead to larger cellular responses ${ }^{1,15}$ at the low concentrations of these agonists that prevail at developing neuromuscular synapses ${ }^{16}$.

There are $\sim 250$ side chain differences between the adult $\varepsilon$ - and fetal $\gamma$-subunits. Mutational correlates of differences between the fetal and adult AChR function have been investigated previously. The mice expressing AChRs having an $\varepsilon-\gamma$-subunit chimera joined at a conserved glycine in loop E, $\gamma(\leq 113)+$ $\varepsilon(\geq 114)$, have a fetal-like open-channel lifetime and adult-like conductance ${ }^{7}$. Synapses expressing these AChRs undergo normal endplate differentiation but show altered innervation patterns. In

a

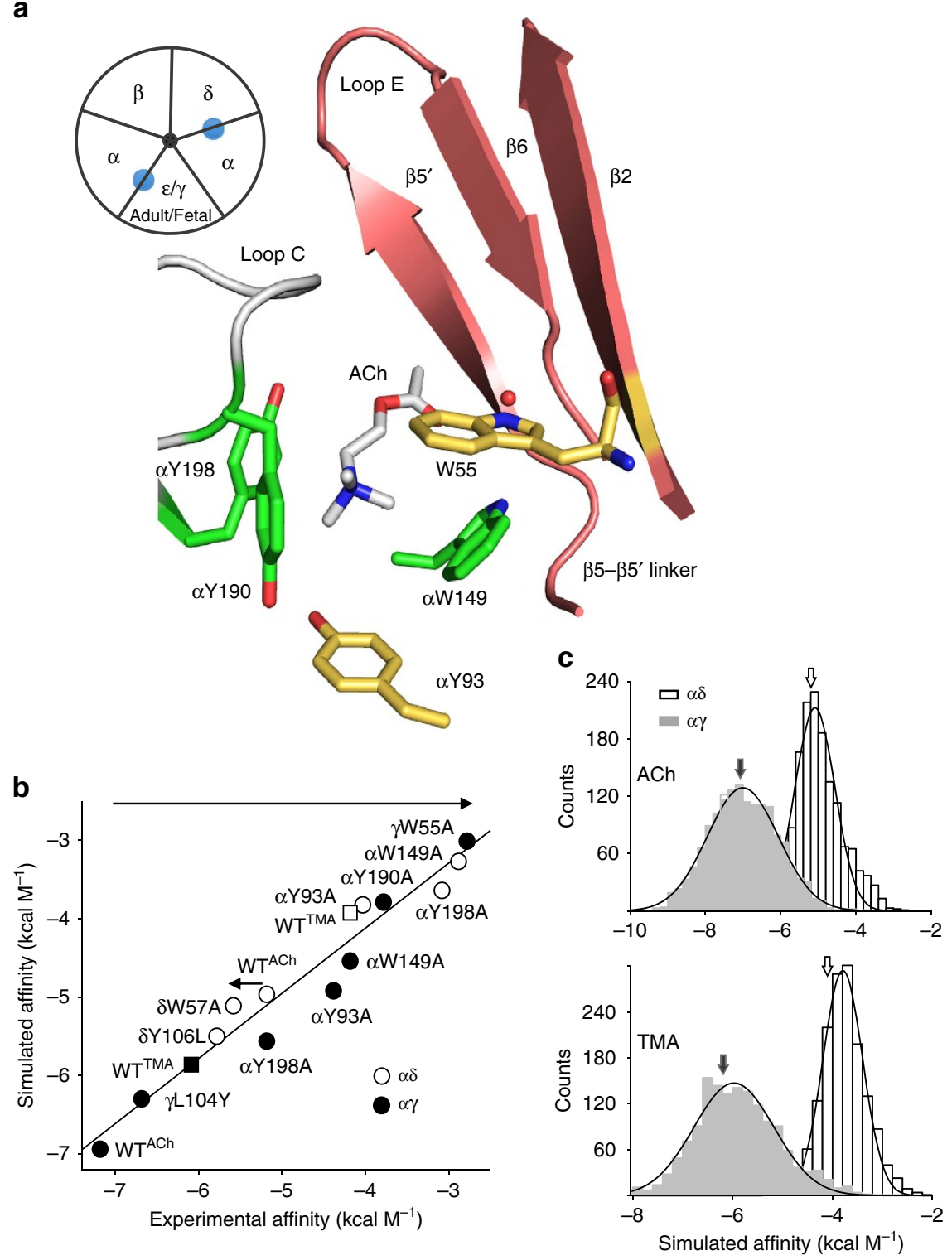

Figure 1 | Ligand binding site and affinities. (a) The principal subunit ( $\alpha$ in AChRs) is left (white) and the complementary subunit ( $\delta$, $\varepsilon$ or $\gamma$ ) is right (pink; Lymnaea stagnalis, pdb accession number 3 WIP ${ }^{27}$ ). Aromatic triad, green; special pair, yellow; structural water, red sphere. Inset: in endplate AChRs only two of the five subunit interfaces are functional binding sites (blue). (b) Affinity estimates from simulations and electrophysiology experiments are correlated. Circles, ACh (alanine mutations of core aromatics except where marked); squares, TMA (WT only). In both simulations and electrophysiology experiments, there is a large loss in binding energy with $\gamma \mathrm{W} 55 \mathrm{~A}$ and a small gain with $\delta \mathrm{W} 57 \mathrm{~A}$ (arrows). (c) Distributions of binding energies from WT AChR simulations. Arrows mark the corresponding in vitro affinities estimated from electrophysiology experiments. 
mouse AChRs, the M3-M4 linker has been shown to influence fetal versus adult open-channel lifetime ${ }^{17}$. In Xenopus AChRs, residues at positions $6^{\prime} / 7^{\prime}$ in $\mathrm{M} 2(\mathrm{~N} / \mathrm{I}$ in $\gamma$ versus $\mathrm{S} / \mathrm{V}$ in $\varepsilon)$ were implicated in setting fetal versus adult conductance ${ }^{18}$, and in rat AChRs, swapping the $\mathrm{M} 2-20^{\prime}$ amino acid from $\mathrm{K}$ to $\mathrm{Q}$ results in a partial exchange of conductance ${ }^{19}$. None of the above studies investigated the structural basis of fetal versus adult affinity, which is the topic we address here.

At all three kinds of agonist site $(\alpha \delta, \alpha \varepsilon$ and $\alpha \gamma)$, ACh is stabilized in the binding pocket by a core of five aromatic residues (Fig. 1a). On the basis of their individual contributions to affinity, these can be divided into two working groups, an 'aromatic triad' and a 'special pair'20. The triad is $\alpha \mathrm{W} 149$ (indole ring), $\alpha \mathrm{Y} 198$ (benzene ring) and $\alpha \mathrm{Y} 190$ (benzene ring and hydroxyl). The four functional groups of these three amino acids each make a similar contribution to ACh binding energy at all the three agonist sites $^{15,21}$. In adult AChRs, these groups provide almost all of the neurotransmitter binding energy, which is equal to $+0.59 \ln K_{\mathrm{d}}$ $\left(23^{\circ} \mathrm{C}\right)$. Together, these groups stabilize ACh by approximately $-5.1 \mathrm{kcal} \mathrm{M}^{-1}$ per site $\left(-10.2 \mathrm{kcal} \mathrm{M}^{-1}\right.$ for $\alpha \delta+\alpha \varepsilon$ sites combined). The action of the triad is led by $\alpha \mathrm{Y} 190$, because the ring and $-\mathrm{OH}$ each contribute approximately $-2 \mathrm{kcal} \mathrm{M}^{-1}$ at $\alpha \delta, \alpha \varepsilon$ and $\alpha \gamma$.

A second working part of the agonist site apparatus is the special pair, $\alpha \mathrm{Y} 93$ (benzene ring) and $\varepsilon / \gamma \mathrm{W} 55$ ( $\delta \mathrm{W} 57$; indole ring). At $\alpha \varepsilon$ and $\alpha \delta$, these two groups make only a small contribution to ACh affinity, but at the fetal $\alpha \gamma$ site, the aromatic rings contribute significantly to bring the single-site-total to approximately $-7.1 \mathrm{kcal} \mathrm{M}^{-1}$ and the $\alpha \delta+\alpha \gamma$ total to $-12.2 \mathrm{kcal} \mathrm{M}^{-1}$ (ref. 15). Moreover, at $\alpha \gamma$, the effects of alanine substitutions of this pair are not independent, as removing either ring interferes substantially with the other's ability to stabilize ACh. The energy contribution of this tryptophan differs massively between the fetal and adult sites. At $\alpha \gamma$, the $\gamma$ W55A mutation decreases affinity by $\sim 2,000$-fold, whereas the homologous substitution at $\alpha \varepsilon$ reduces it by only $\sim 13$-fold and at $\alpha \delta$ it actually increases affinity by $\sim 2$-fold.

Because the aromatic core has the same composition at all the three kinds of agonist sites, the difference in affinity between the fetal and adult AChRs can be attributed to residues in the complementary, non- $\alpha$-subunit $(\delta, \varepsilon$ or $\gamma$ ). Our approach to finding these amino acids was to use molecular dynamics (MD) simulations of homology models based on AChBP to identify complementary-subunit amino acids near the core that influence the contribution of the special pair to affinity. Then, we exchanged those side chains in vitro $(\delta$ and $\varepsilon \leftrightarrow \gamma)$ and estimated affinity from single-channel currents of AChRs expressed in cells. The results indicate that four residues in combination determine adult versus fetal resting affinity, three in loop E (111-113; $\gamma$-subunit numbers) and one in the $\beta 5-\beta 5^{\prime}$ linker (104). In the $\beta 5^{\prime}-\beta 6$ hairpin region, mutations of the human $\gamma$-subunit (V108, S111, P112 and P121) cause multiple pterygium and Escobar syndromes ${ }^{12}$. The simulations suggest that these amino acids influence core properties by changing the structure and dynamics of the $\beta 5-\beta 5^{\prime}$ linker and the complementary $\beta$-sheet, to effect the action of the special pair and the affinity for the agonist. Swapping the four side chains is both necessary and sufficient to exchange fetal versus adult affinities and open-channel lifetimes.

The results are presented in five sections. First, we build and test homology models for the fetal and adult agonist sites. Second, we use the models to identify residues in the complementary subunit that influence ACh affinity in silico. Third, we use electrophysiology to measure in vitro affinities of AChRs having the identified residues swapped, fetal $\leftrightarrow$ adult. Fourth, we report the effects of point mutations on affinity, from the identified group and of a critical, complementary-side tryptophan. Fifth, we analyse the simulated structures and develop hypotheses for the mechanisms that undergird the fetal versus adult affinity difference.

\section{Results}

Tuning the model. Our approach was to use MD simulations to guide mutagenesis, and electrophysiology experiments to measure in vitro affinities. We used an empirical and computationally inexpensive method of estimating agonist binding energy in silico (see Methods section). By adjusting the free parameters for van der Waals and electrostatic contributions in the calculations, simulated and experimental affinities could be correlated (Fig. 1b). This was possible, in part, because in AChRs the entropy component of affinity is small ${ }^{22,23}$.

The circles in Fig. $1 \mathrm{~b}$ are simulated versus experimental ACh binding energies for $\alpha \gamma$ and $\alpha \delta$ agonist sites with mutations of core residues. The free parameters in the binding energy calculation (Equation 1, Methods section) were obtained only from the alanine mutations. Two non-alanine substitutions fell on the same regression line. The arrows indicate that the large and opposite effect of an alanine substitution of the complementary tryptophan on affinity at $\alpha \gamma$ versus $\alpha \delta$ that is apparent in vitro was reproduced in silico. Also, in both simulations and experiments the $\alpha \gamma^{\mathrm{WT}}$ site showed $\sim 40 \%$ more favourable binding energy for $\mathrm{ACh}$ and tetramethylammonium (TMA) compared with the $\alpha \delta^{\mathrm{WT}}$ site (Fig. 1c). These results suggest that brief simulations and approximate binding energy estimates can be used to screen for amino acids that influence affinity.

C4 in silico. The first goal was to identify residues in the complementary, $\gamma$-versus $\delta$-subunit that are responsible for the 30 fold higher affinity for ACh in $\alpha \gamma$ versus $\alpha \delta$. We used two criteria to select candidate residues to be exchanged in silico. We chose side chains that in the model were within $20 \AA$ of the quaternary ammonium (QA) group of ACh and were different between $\gamma$ and $\delta$ but homologous between $\varepsilon$ and $\delta$. A total of 10 amino acids satisfied both of these criteria (C10; Fig. 2a).

In the first set of simulations, all the $\mathrm{C} 10$ side chains were swapped, $\gamma \rightarrow \delta$ and $\delta \rightarrow \gamma$, and ACh binding energies were calculated. The results were that the C10-mutated $\alpha \delta$ site provided about the same binding energy as the $\alpha \gamma^{\mathrm{WT}}$ site, and the C10-mutated $\alpha \gamma$ site provided about the same as the $\alpha \delta^{\mathrm{WT}}$ site (Fig. 2b). This suggested that the mutation(s) we were seeking were within the $\mathrm{C} 10$ group.

We then winnowed the mutation list. First, each member of the C10 group was dropped one at a time. The result of these nineswap simulations was that the affinities no longer reversed when one of 4 of the original 10 amino acids was omitted. When $\gamma(\mathrm{L} 104, \mathrm{~S} 111, \mathrm{P} 112$ or D113) or $\delta(\mathrm{Y} 106, \mathrm{Y} 113, \mathrm{D} 114$ or S115) side chains remained as in the parent subunit, the full affinity reversal observed for $\mathrm{C} 10$ was incomplete. We call this set of four complementary subunit side chains $\mathrm{C} 4$.

Next, we simulated affinities using models in which only the C4 amino acids were exchanged as a group, to make constructs $\alpha \gamma^{\mathrm{C} 4 \delta}$ and $\alpha \delta^{\mathrm{C} 4 \gamma}$ (regular text is parent and superscript is target). Swapping all C4 side chains was sufficient to make the $\alpha \gamma$ site have an affinity like $\alpha \delta^{\mathrm{WT}}$ and the $\alpha \delta$ site to have an affinity like $\alpha \gamma \mathrm{WT}$. In silico, exchanging the $\mathrm{C} 4$ set of side chains was sufficient to exchange affinities, stably (Fig. 2b).

In the next set of simulations, each of the six other residues of the C10 starting set was added to C4, one at a time. None of these made a significant difference in the magnitude of the affinity swap. Finally, we dropped each mutation from the $\mathrm{C} 4$ set, one at a time, and estimated ACh affinity. Unlike in the above simulations, the trajectories of these three-mutant swaps were not 
a
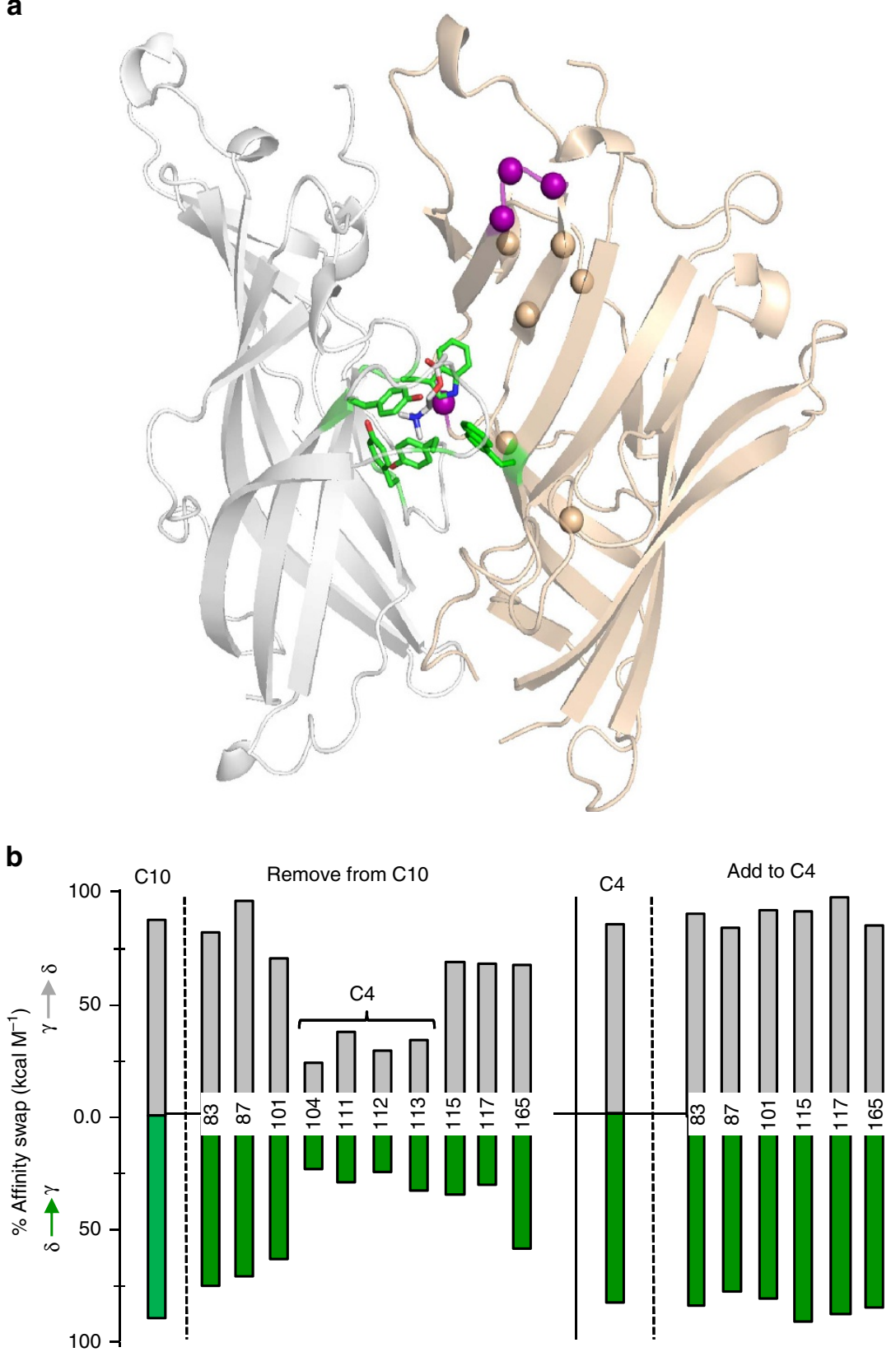

Figure 2 | Simulations identify affinity-influencing side chains. (a) Ten residues in the complementary subunit (tan) are within $20 \AA$ of the ACh quaternary amine and are different in $\gamma$ versus $\delta \approx \varepsilon$ (large spheres; C10). Core, green; C4 residues, violet. (b) Affinity changes calculated in silico for different combinations of side-chain swaps. Residue numbers pertain to the $\gamma$-subunit. Left-to-right: swapping all C10 residues almost completely exchanges affinity; dropping any one of four residues from C10 reduces the magnitude of the affinity change (C4; violet spheres in a); swapping all C4 side chains together is sufficient to exchange affinity; adding residues does not improve significantly the effect of the C4 swap.

stable (both fluctuating and divergent root mean square deviation; Supplementary Fig. 1). In silico, C4 was the minimum construct required for exchanging stably $\alpha \gamma$ - and $\alpha \delta$-binding energies.

C4 in vitro. We next made corresponding experimental measurements of ACh affinity $\left(K_{\mathrm{d}}\right)$ from single-channel currents recorded from AChRs expressed in HEK cells (Fig. 3). The C4 residues were investigated at $\alpha \gamma-, \alpha \delta$ - and $\alpha \varepsilon$-binding interfaces. In these electrophysiology experiments, the constructs were $\alpha \delta^{\mathrm{C} 4 \gamma}$ $(\delta \mathrm{Y} 106 \mathrm{~L}+\delta \mathrm{Y} 113 \mathrm{~S}+\delta \mathrm{D} 114 \mathrm{P}+\delta \mathrm{S} 115 \mathrm{D}), \quad \alpha \varepsilon^{\mathrm{C} 4 \gamma} \quad(\varepsilon \mathrm{Y} 104 \mathrm{~L}+$ $\varepsilon \mathrm{Y} 111 \mathrm{~S}+\varepsilon \mathrm{E} 112 \mathrm{P}+\varepsilon \mathrm{G} 113 \mathrm{D})$ and $\alpha \gamma^{\mathrm{C} 4 \delta}(\gamma \mathrm{L} 104 \mathrm{Y}+\gamma \mathrm{S} 111 \mathrm{Y}+$ $\gamma \mathrm{P} 112 \mathrm{D}+\gamma \mathrm{D} 113 \mathrm{~S})$.

First, the in vitro measurements were made using AChRs that had only one functional binding site, its companion being knocked out by a mutation(s) ${ }^{24}$. The results were that the $\mathrm{C} 4 \varepsilon \rightarrow \gamma$ and $\delta \rightarrow \gamma$ exchanges resulted in a nearly complete swap of affinity (Fig. 3a). The $\alpha \varepsilon^{\mathrm{C} 4 \gamma}$ and $\alpha \delta^{\mathrm{C} 4 \gamma}$ constructs each had the doseresponse profile and affinity of the target, $\alpha \gamma \mathrm{WT}$ site. The $K_{\mathrm{d}}^{\mathrm{ACh}}$ values for $\alpha \varepsilon^{\mathrm{C} 4 \gamma}$ and $\alpha \delta^{\mathrm{C} 4 \gamma}$ are shown in Supplementary Table 1.

The behaviour of $\alpha \gamma^{\mathrm{C} 4 \delta}$ was more complex (Fig. 3b). This construct alternated between two distinct modes of single-channel activity. One mode retained the high-affinity 

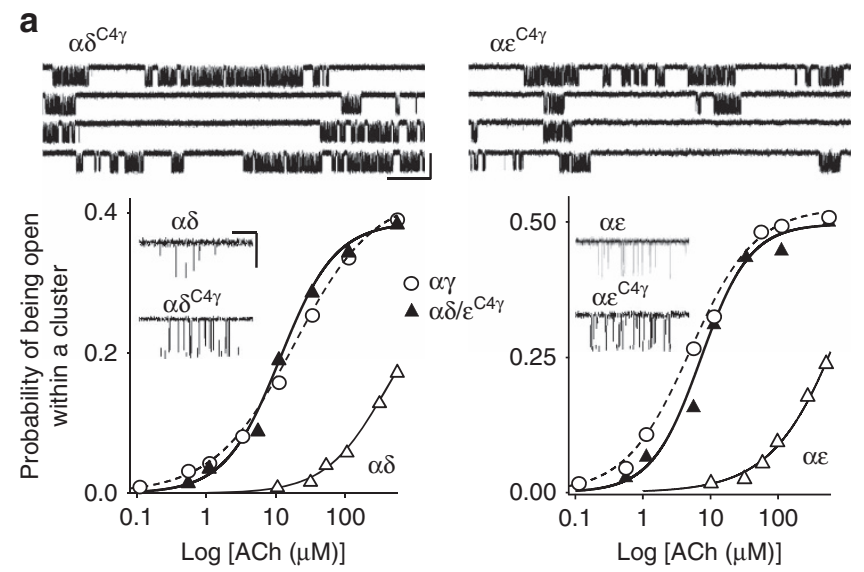

b

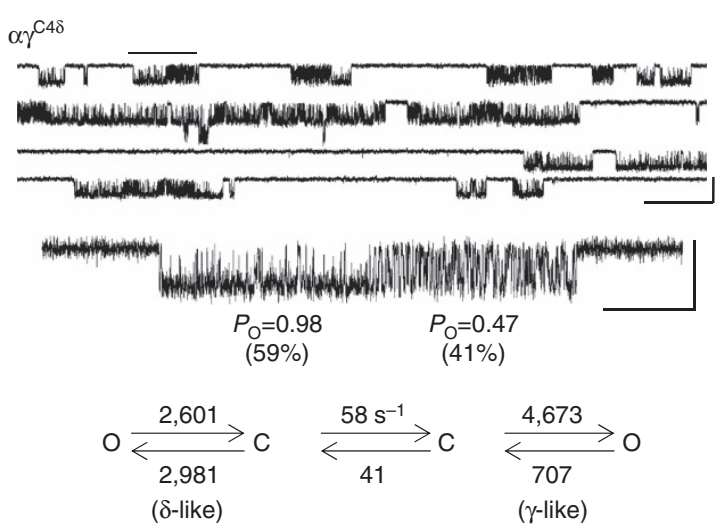

Figure 3 | Electrophysiology measurements corroborate simulations.

(a) Replacing all of the $\delta / \varepsilon$ C4 side chains with those of $\gamma\left(\alpha \delta / \varepsilon^{C 4 \gamma}\right)$ fully exchanges ACh affinity. In all traces, openings are down. Top, low timeresolution view of single-channel currents (scale bar: vertical, $4 \mathrm{pA}$; horizontal, $0.5 \mathrm{~s}$ ). The long gaps between clusters of openings are sojourns in desensitized states; affinity was estimated from the intra-cluster interval durations; membrane potential was $+70 \mathrm{mV}$ to reduce channel block by ACh $(100 \mathrm{mM})$; only one agonist site was functional. Bottom, dose-

response curves. Inset, example clusters $\left(10 \mu \mathrm{M} \mathrm{ACh}, V_{\mathrm{m}}=-100 \mathrm{mV}\right.$, no channel block, scale bar: vertical, $7 \mathrm{pA}$; horizontal, 0.1s). (b) Top, replacing all of the $\gamma \mathrm{C} 4$ side chains with those of $\delta\left(\alpha \gamma^{C 4 \delta}\right)$ generates AChRs that switch between fetal and adult affinity and open-channel lifetime $(100 \mathrm{mM}$ ACh, $V_{m}=+70 \mathrm{mV}$, scale bar: vertical, $4 \mathrm{pA}$; horizontal, $0.5 \mathrm{~s}$ ). Bottom, bimodal kinetics of single-channel intra-cluster interval duration (scale bar: vertical, 4 pA; horizontal, $50 \mathrm{~ms}$ ) showing the $\alpha \gamma \leftrightarrow \alpha \delta$ switch in behaviour.

(Supplementary Table 1) and longer open-channel lifetime characteristic of the parent $\left(\alpha \gamma^{\mathrm{WT}}\right)$ and the other had the lowaffinity and briefer open-channel lifetime characteristic of the target $\left(\alpha \delta^{\mathrm{WT}}\right)$. The prevalence of each mode was approximately equal (isoenergetic), with a switching time constant of $\sim 20 \mathrm{~ms}$. The $C 4 \gamma \rightarrow \delta$ affinity exchange in silico was stable, but in vitro the swap in binding and gating functions was bimodal.

In addition to decreasing the $\mathrm{ACh}$ equilibrium dissociation constant (increasing affinity), the $\alpha \varepsilon / \delta^{\mathrm{C} 4 \gamma}$ mutations generated a fetal-like, slower channel-closing rate constant in the presence of the neurotransmitter ACh. The effects of these $\mathrm{C} 4 \gamma$ mutations in the absence of agonists or in the presence of saturating [ACh] are shown in Fig. 4a,b, with the results summarized in Fig. 5a and Supplementary Table 1 . The C4 substitutions were effective in exchanging ACh affinity. We also measured affinity for the partial agonists carbamylcholine (CCh), TMA and choline and these exchanged affinity partially with $\mathrm{C} 4$ mutations. On average, the low $\rightarrow$ high affinity C4 swaps were $\sim 90 \%$ effective and the high $\rightarrow$ low affinity swaps were $\sim 70 \%$ effective.

We also made in vitro measurements from AChRs having only three of the $\mathrm{C} 4$ side-chain exchanges. The kinetic properties of these $\mathrm{C} 3$ receptors were complex and we did not attempt to estimate affinity.

The two WT AChR agonist sites act nearly independently in so far as binding energies measured from two sites combined are approximately the sums of single-site energies ${ }^{15}$. In the next set of experiments, we examined in vitro two different mutated, adult AChRs (both sites functional), $\alpha \varepsilon^{\mathrm{C} 4 \gamma}+\alpha \delta^{\mathrm{WT}}$ and $\alpha \varepsilon^{\mathrm{C} 4 \gamma}+\alpha \delta^{\mathrm{C} 4 \gamma}$. The prediction was that the first would have a fetal-type affinity and the second would be a 'super' AChR having the high, fetaltype affinity at both agonist sites. These expectations were confirmed, approximately (Fig. 5b,c).

Point mutations. The effects of point mutations of each C4 residue at each agonist site are shown in Fig. 5d. In experiments, none of these single exchanges had a large $\left(>1 \mathrm{kcal} \mathrm{M}^{-1}\right)$ effect on affinity or gating without agonists. With regard to affinity, the sum of the $\delta \rightarrow \gamma$ single-residue C4 swaps was $-2.8 \mathrm{kcal} \mathrm{M}^{-1}$, which is close to that for the $\alpha \delta^{\mathrm{C} 4 \gamma}$ combination, but the sum of the $\gamma \rightarrow \delta$ single-residue swaps was only $\sim+0.5 \mathrm{kcal} \mathrm{M}^{-1}$. With regard to the allosteric constant, none of the $\mathrm{C} 4$ swap sums were sufficient to account for the $\sim 14$-fold smaller unliganded gating equilibrium constant $\left(+1.6 \mathrm{kcal} \mathrm{M}^{-1}\right)$ apparent in fetal versus adult AChRs at $-100 \mathrm{mV}$ (ref. 1).

The mouse AChR $\varepsilon-\gamma$ chimera was joined at a conserved glycine in loop $\mathrm{E}^{7}$. We were unable to record single-channel currents from AChRs having a point substitution here ( $\gamma$ G114 to $\mathrm{A}, \mathrm{L}$ or $\mathrm{P}$ ) because these receptors either failed to express or open.

The action of the complementary, special pair tryptophan is the main distinction between $\alpha \gamma$ versus $\alpha \delta$ sites ${ }^{15,25,26}$. The huge difference in the effect of an alanine substitution at W55 in $\gamma$ versus $\delta$ in vitro was also apparent in silico (Fig. 1b). To further test both the model and the ability of $\mathrm{C} 4$ to swap the properties of the aromatic core, we compared simulated and experimental ACh binding energies for the $\mathrm{C} 4$ constructs in the presence of the point mutation $\gamma / \varepsilon W 55 \mathrm{~A}$ or $\delta \mathrm{W} 57 \mathrm{~A}$. If C4 was completely responsible for the different contributions of the special pair, we expected that an alanine substitution would have either no or little effect at $\alpha \gamma^{\mathrm{C} 4 \delta / \varepsilon}$ (as in $\alpha \delta / \varepsilon^{\mathrm{WT}}$ ), but would cause a large decrease in affinity at $\alpha \delta / \varepsilon^{\mathrm{C} 4 \gamma}$ (as in $\alpha \gamma^{\mathrm{WT}}$ ). The results agreed with these predictions, but partially (Fig. 6). We did not test $\alpha \gamma^{\mathrm{C} 4 \delta}(+\delta \mathrm{W} 55 \mathrm{~A})$ in electrophysiology experiments because $\alpha \gamma^{\mathrm{C} 4 \delta}$ gave rise to complicated, heterogeneous responses without the tryptophan mutation (Fig. 3).

Analyses of structures. The modelling and electrophysiology results suggest that the $\beta$-sheet of the complementary surface of the agonist site can fold stably into, or isomerize between (Fig. 3b), either of two approximately isoenergetic configurations to produce a high, $\gamma$-like or a low, $\delta$-like resting affinity (HA or LA, not to be confused with active-state vs resting-state affinities). Further, they suggest that the $\mathrm{C} 4$ side chains bias which of these super-secondary structures is adopted. Because there are no atomic-resolution structures of fetal or adult AChR agonist sites with agonist bound, we analysed structures generated in the MD simulations to explore possible difference between the alternate conformations.

The two regions of interest were the core and the complementary $\beta$-sheet. Figure 7 shows representative snapshots from C4-swapped trajectories and Fig. 8a shows quantitative analyses of the HA versus LA structures. The structural parameters 
a

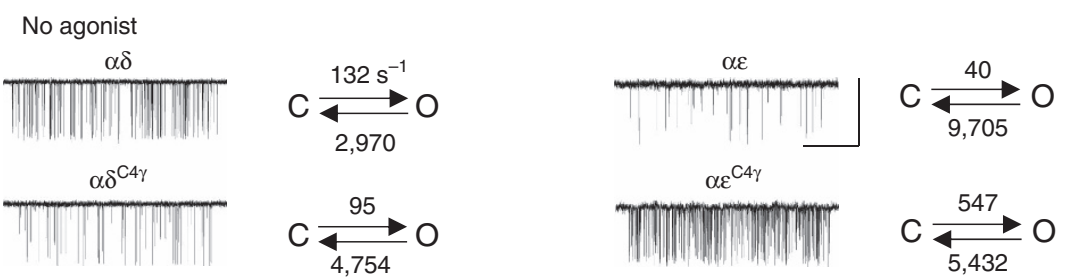

b One-site occupied by ACh
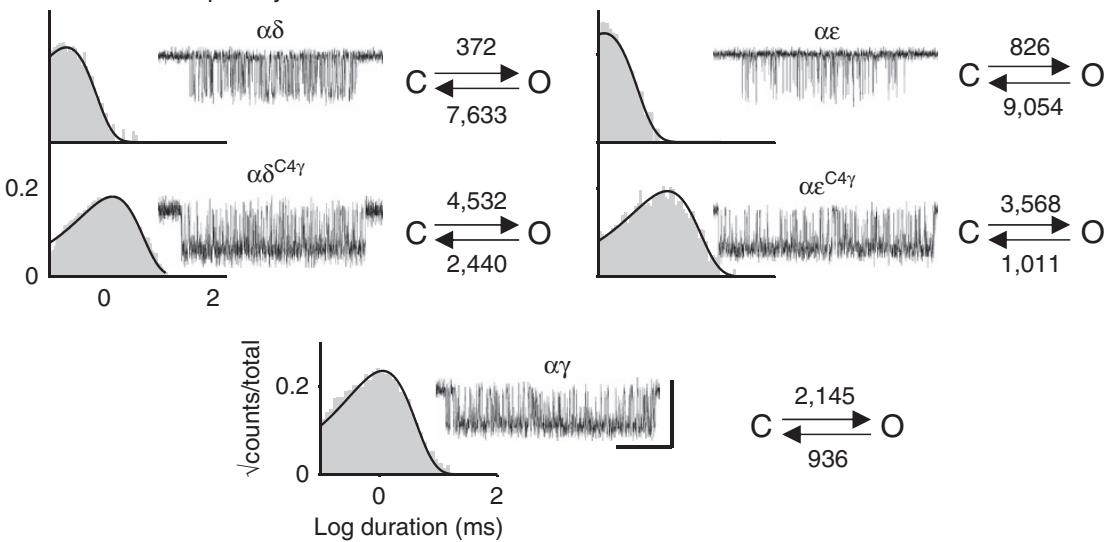

Figure 4 | C4 mutations swap fetal versus adult gating kinetics. (a) Gating without agonists (openings are down; $V_{m}=-100 \mathrm{mV}$, scale bar: vertical, $7 \mathrm{pA}$; horizontal, $250 \mathrm{~ms}$ ). At $\alpha \delta$ and $\alpha \varepsilon$, the effects of the $C 4 \gamma$ mutations are $<2$-fold except for the $\alpha \varepsilon$ opening rate constant. C4 mutations have a small effect on unliganded gating. (b) Gating with one functional agonist binding site occupied by $\mathrm{ACh}\left(100 \mathrm{mM} ; V_{\mathrm{m}}=+70 \mathrm{mV}\right.$ to reduce channel block, scale bar: vertical, $4 \mathrm{pA}$; horizontal, $50 \mathrm{~ms}$ ). At $\alpha \delta$ and $\alpha \varepsilon$, the $C 4 \gamma$ mutations cause the channel-closing rate constant to become like that of the target subunit. Histograms are open-channel lifetime distributions. See Fig. 5a for summary.

a
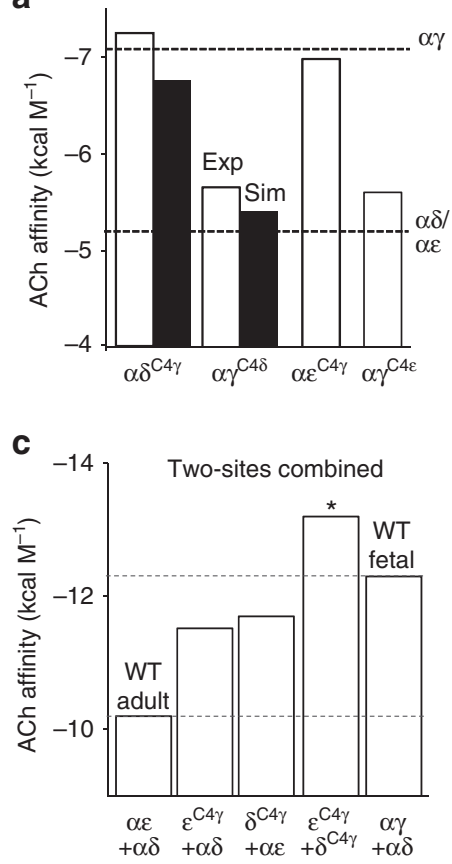

b

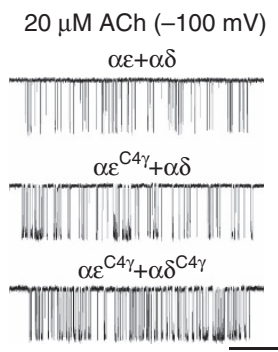

d

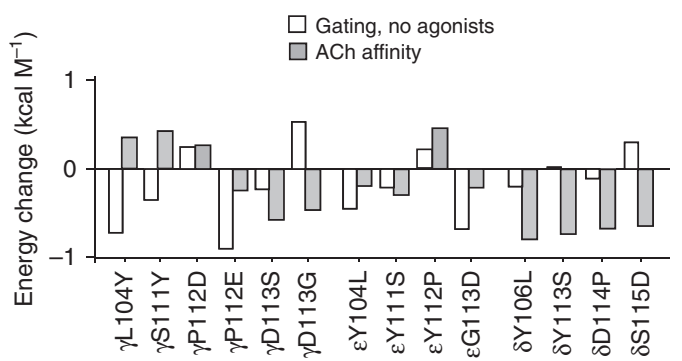

Figure 5 | ACh affinities for one- and two-site AChRs. (a) ACh affinities ( +0.59 InK ${ }_{d}$ ) of C4-swapped constructs in AChRs having a single functional agonist binding site (exp, from electrophysiology; sim, from MD simulations; see Supplementary Table 1). Dashed lines, WT affinities measured by using electrophysiology ${ }^{15}$. The C4 mutations exchange affinity. (b) Swapping C4 at both adult sites $(\alpha \varepsilon+\alpha \delta)$ in two-site AChRs. Example single-channel currents (openings are down, scale bar: vertical, $7 \mathrm{pA}$; horizontal, $50 \mathrm{~ms}$ ). (c) Total ACh binding energy from both sites combined; ${ }^{\star}$, the doubly swapped adult AChR $\left(\varepsilon^{C 4 \gamma}+\delta^{C 4 \gamma}\right)$ has even-more favourable ACh binding energy than the WT fetal AChR. (d) Effects of swapping C4 side chains one at a time in one-site receptors. 
a

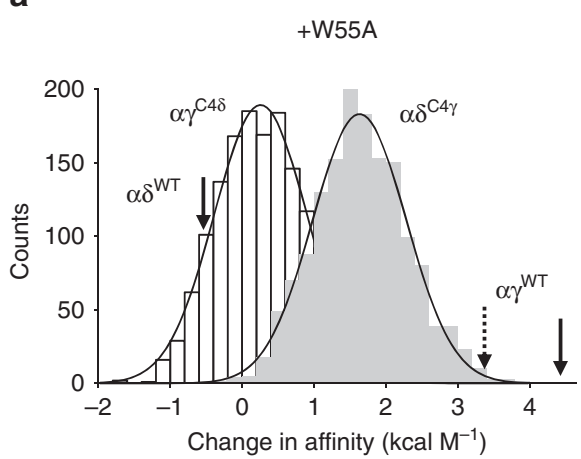

b

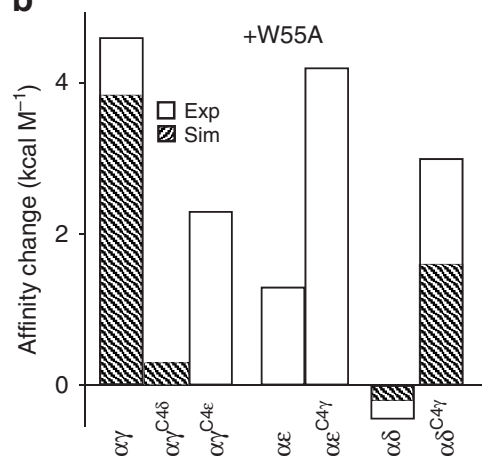

Figure 6 | The action of W55 in C4-swapped constructs. (a) Change in ACh affinity with an added W55A mutation. Histograms simulated from C4-swapped constructs; solid arrows, electrophysiology results in WT background (see Fig. 1b). Left, for the $\alpha \gamma^{\mathrm{C} 4 \delta}$ and $\alpha \delta^{\mathrm{WT}}$ backgrounds, the change in affinity was similar and small in both simulations and experiments. Right, for the $\alpha \delta^{\mathrm{C} 4 \gamma}$ and $\alpha \gamma^{\mathrm{WT}}$ backgrounds, the simulated change in affinity was smaller than observed in experiments. Dashed line, simulated change in affinity for the $\alpha \gamma^{W T}$ background. (b) Summary of W55A mutational effects on ACh affinity. In both the simulations and experiments, the energy change was partial for $\alpha \gamma^{\mathrm{C} 4 \delta}$ and $\alpha \delta^{\mathrm{C} 4 \gamma}$ but nearly complete for $\alpha \varepsilon^{\mathrm{C} 4 \gamma}$.
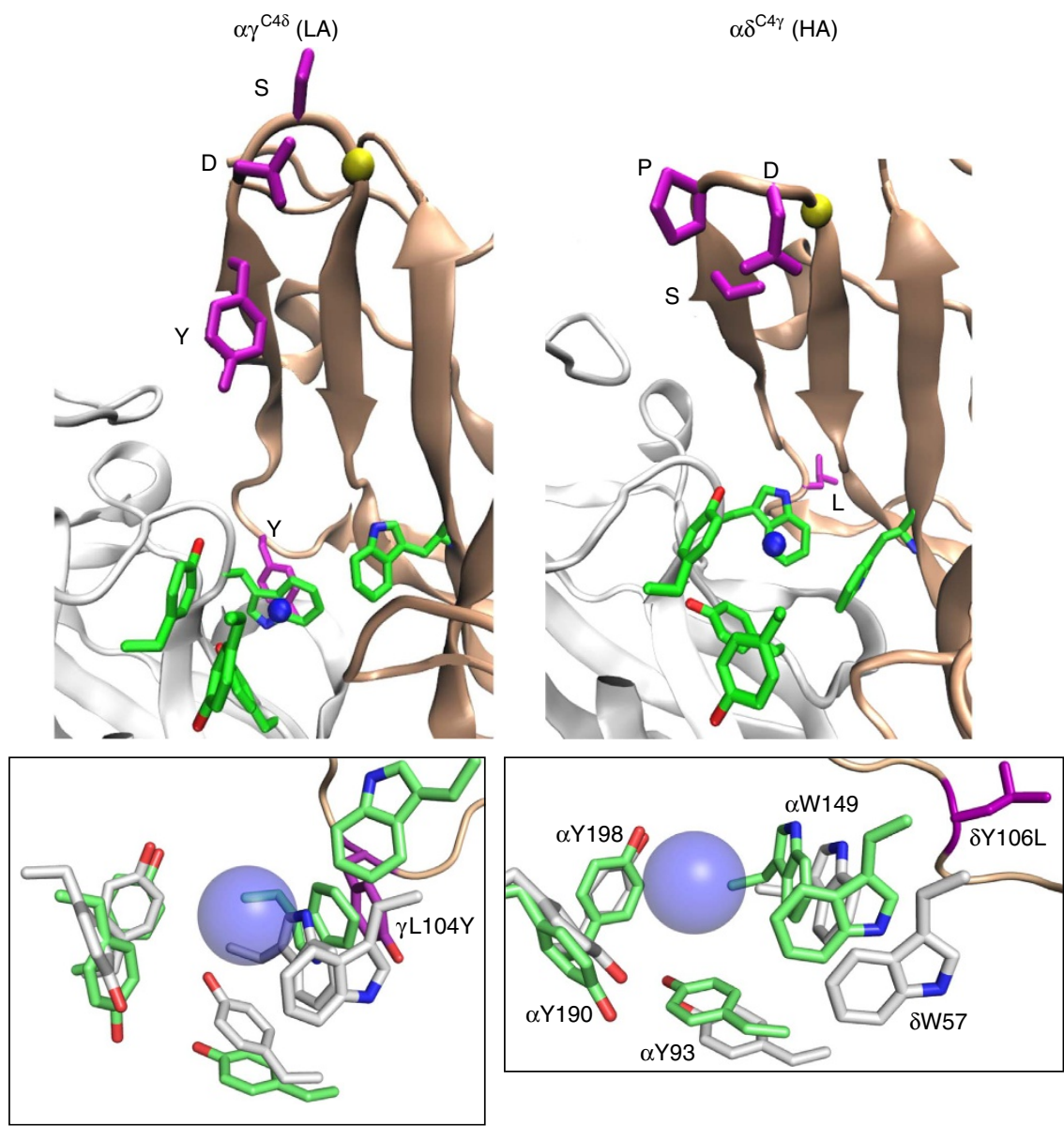

Figure 7 | Representative snapshots of C4-swapped constructs. The snapshots have the lowest RMSD from the mean. Top: Low-resolution views; $\alpha$-subunit, white; complementary subunit, tan; core aromatics, green; C4 amino acids, violet; ACh nitrogen, blue sphere; $\alpha \mathrm{C}$ of conserved loop E glycine, yellow. Bottom: high-resolution view of core in the parent (white) and C4-swapped (green) constructs. In the higher affinity (HA) structures, the binding pocket is more compact and W55 is closer to agonist (quaternary ammonium group, large blue sphere).

estimated from C4-swapped constructs were similar to those from the parent subunits of corresponding affinity.

Representative snapshots of the core from $\alpha \gamma^{\mathrm{C} 4 \delta}$ and $\alpha \delta^{\mathrm{C} 4 \gamma}$ trajectories are shown in Fig. 9a. In LA versus HA structures, the $\beta 5^{\prime}$ linker residue Y104 is close and face on with $\alpha \mathrm{W} 149$, and $\beta 2$ residue W55 is distant and not orthogonal to either $\alpha \mathrm{W} 149$ or $\alpha \mathrm{Y} 93$. The structural differences of the $\alpha$-subunit aromatic triad were less pronounced, but in the LA structures these side chains 

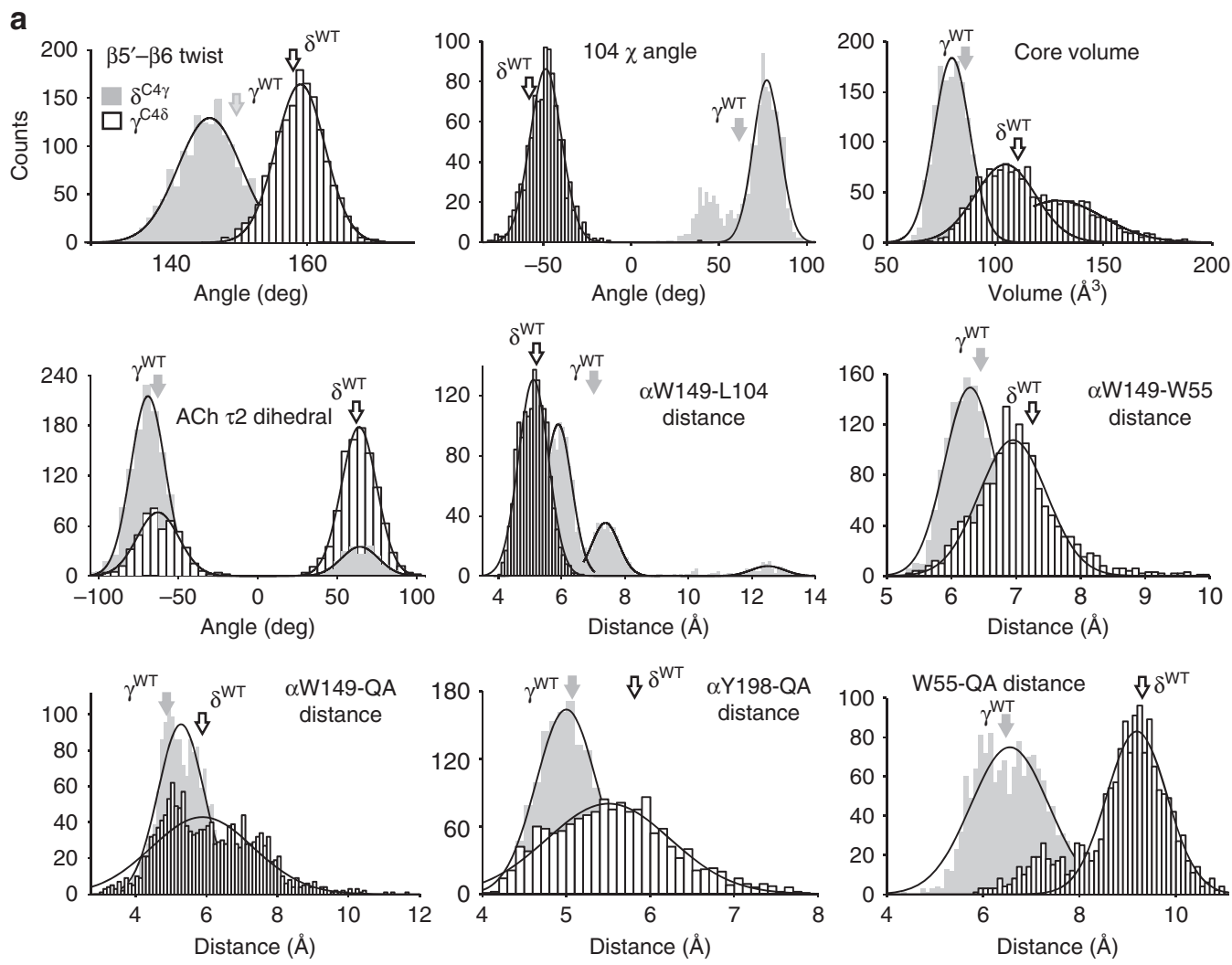

b

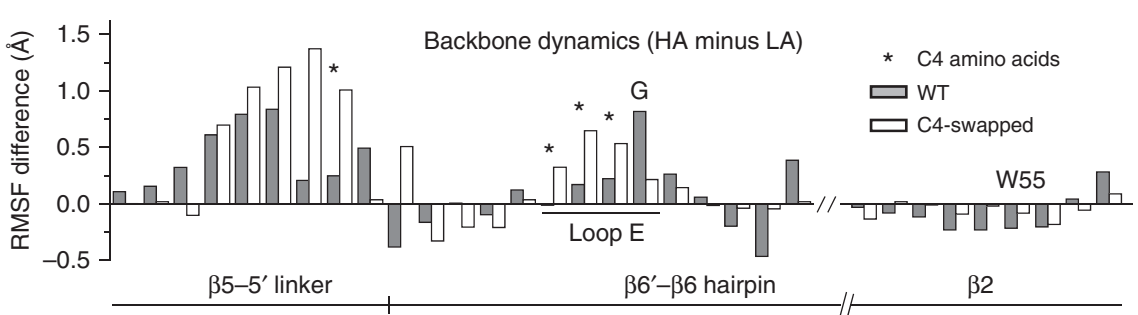

Figure 8 | Quantitative analyses of MD results. (a) Structural parameters estimated from trajectories. Histograms are from C4-swaps; arrows are mean values from WT trajectories. (b) HA RMSF minus LA. WT, grey ( $\alpha \gamma$ minus $\alpha \delta$ ) and C4-swap, white $\left(\alpha \delta^{\mathrm{C} 4 \gamma}\right.$ minus $\left.\alpha \gamma^{\mathrm{C} 4 \delta}\right)$. The $\beta 5-\beta 5^{\prime}$ linker and loop E are more flexible in HA structures, whereas the base of the hairpin and $\beta 2$ near W55 are more flexible in LA structures (see Fig. 9c).

were all further from the agonist's quaternary amine (QA). Overall, the simulations indicate that the HA core is more compact, organized and stable.

In AChBP there is a structural water within the core that is $\mathrm{H}$-bonded to $\mathrm{ACh}$ and the backbones of $\beta 5^{\prime}$ and $\beta 6^{23,27,28}$ (Fig. 1a). In the simulations, both this water and ACh were dynamic (Fig. 9c). The water- $\beta 6 \mathrm{H}$-bond had the same propensity in both HA and LA structures, but the H-bonds with ACh and $\beta 5$ were less prevalent in LA trajectories (Supplementary Table 2). In the simulations, the $\mathrm{N}-\mathrm{C}-\mathrm{C}-\mathrm{O}$ dihedral angle $(\tau 2)$ of the neurotransmitter was bimodal, being either $-60^{\circ}$ or $+60^{\circ}$ (Figs $8 \mathrm{a}$ and $9 \mathrm{a}$ ). The $+60^{\circ}$ configuration was approximately five times more common in LA trajectories.

We also estimated structural parameters for the complementary surface, which is made up of the $\beta 5^{\prime}$ linker, the $\beta 5^{\prime}-\beta 6$ hairpin (that includes loop E) and strand $\beta 2$ (Fig. 1a). Figure 9b (top) shows inter-strand backbone $\mathrm{H}$-bonds for the hairpin. In LA versus HA structures, loop $\mathrm{E}$ had three versus four residues, because with the HA C4 amino acids (SPD), the pre-proline backbone $111(\mathrm{O})$ is rotated out of the hairpin plane and fails to form an H-bond with the post-glycine backbone 115(N)
(Supplementary Table 2). With LA amino acids (YDS), the 111-115 amide-oxygen $\mathrm{H}$-bond is present so that the first residue is part of the hairpin rather than the loop. Also, in HA structures, the S111 side chain has an appropriate distance and geometry to H-bond with the loop E backbone (D113 or G114). The other difference in $\mathrm{H}$-bonds is near the base of the hairpin, where the $109(\mathrm{~N})-117(\mathrm{O})$ bond is less prevalent in LA structures. Figure $9 \mathrm{~b}$ (bottom) shows that in the simulations, the overall hairpin is more upright (by $\sim 8^{\circ}$ ) and less twisted (by $\sim 9^{\circ}$; Fig. $8 \mathrm{a}$ ) in LA structures.

There were fewer H-bonds between the W55 backbone and neighbouring residues in LA structures (Fig. 9c and Supplementary Table 2). Although the $\beta 2-\beta 1$ strand bond W55(N)-T36(O) was equally likely in LA and HA structures, the backbone H-bonds between W55(O)-T36(N) and W55(O)E57(N) were less prevalent in LA.

Finally, we used root-mean-square fluctuation (RMSF) analyses to estimate the dynamics of the complementary $\beta$-sheet backbone (Fig. 8b). The results are summarized in Fig. 9c. In LA structures, the RMSF of the $\beta 5^{\prime}$ linker and loop E (that is, all C4 residues) was significantly smaller than in $\mathrm{HA}$ structures. This 

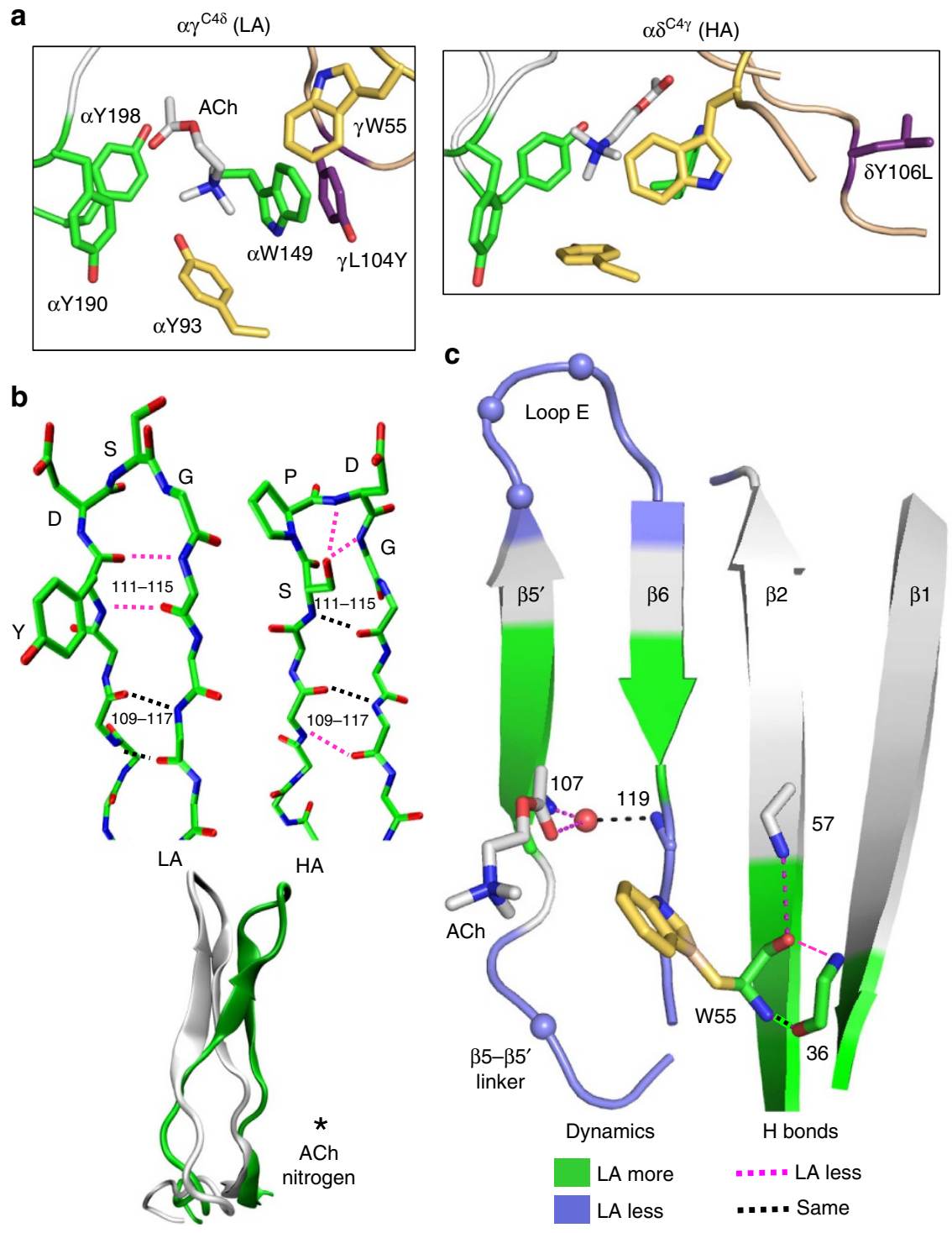

Figure 9 | Structures suggest a mechanism for fetal versus adult affinity. (a) Representative snapshots of C4-swapped cores (LA, low affinity $\alpha \delta^{\mathrm{WT}} / \alpha \gamma^{\mathrm{C} 4 \delta}$ and HA, high affinity $\alpha \gamma^{\mathrm{WT}} / \alpha \delta^{\mathrm{C} 4 \gamma}$ ). Left, the LA core is spread, $\gamma \mathrm{W} 55$ is far from ACh, C4 residue $\gamma \mathrm{L} 104 \mathrm{Y}$ (purple) is face-on to $\alpha \mathrm{W} 149$ and $\mathrm{ACh}$ is bent. Right, the HA core is compact, $\delta \mathrm{W} 57-\alpha \mathrm{W} 149-\alpha Y 93$ are orthogonal, $\delta Y 106 \mathrm{~L}$ points away from the agonist and ACh is straight. (b) H-bonds of the $\beta 5^{\prime}-\beta 6$ hairpin. Top, dotted lines are H-bonds. pink, bonds that correlate with affinity (are $\sim 2 \times$ more prevalent in simulations of LA or HA sites); black, bonds that are equally prevalent in LA and HA trajectories (Supplementary Table 2). Loop E (bold side-chain labels) is one residue shorter in LA structures. Bottom, in LA constructs the hairpin is straighter and less twisted. (c) Relative dynamics of the $\beta$-sheet backbone. LA is associated with a smaller RMSF

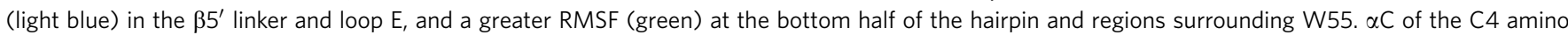
acids, light blue spheres; structural water oxygen, red sphere; H-bonds, dashed lines; numbers are after $\gamma$-subunit. PDB accession number 3WIP; see Fig. 8 and Supplementary Table 2 for quantitative analyses.

relative stability was accompanied by higher dynamics in other regions, in particular in the bottom half of the $\beta$ hairpin and the $\beta 2$ strand in the vicinity of W55. These differences in backbone dynamics correlate with the lower probability of $\mathrm{H}$-bonds in LA structures at the base of the $\beta$-hairpin, with $\beta 5$ and ACh and in $\beta 2$ (Supplementary Table 2). In summary, in the simulations, lower affinity was associated with a straighter hairpin, a lessdynamic $\beta 5^{\prime}$ linker, a shorter and less-dynamic loop $\mathrm{E}$, and a more-dynamic backbone near the core (base of hairpin, structural water and W55).

\section{Discussion}

It was possible to swap in vitro the affinities of the fetal and adult agonist sites based on mutations identified in silico. Apparently, (i) the Aplysia AChBP is a good model of the endplate AChR resting $\alpha \gamma$ agonist site, (ii) classical force fields, short simulation times and approximate estimates of binding energy are adequate and (iii) other structural elements of the biological AChR (transmembrane and intracellular domains, lipid bilayer, posttranslational modifications) are not major determinants of ACh affinity. The results suggest that simulations that are not state-ofthe-art can nonetheless be used effectively as an engineering tool.

The $\mathrm{C} 4$ set of residues determines some of the functional differences between fetal versus adult AChRs. Swapping just these four side chains exchanges fetal versus adult AChR affinities and open-channel lifetimes. This suggests that the complementary, super-secondary $\beta$-sheet can adopt either a fetal-type (HA) or an adult-type (LA) conformation. The C4 mutation set does not, however, account for fetal versus adult differences in the 
unliganded gating equilibrium constant, gating-voltage dependence, single-channel conductance or ion selectivity. Nonetheless, it is now possible to control by C4 mutation(s) an AChR's affinity for the neurotransmitter and, by other mutations the allosteric constant, open-channel lifetime and, to some extent, singlechannel conductance. This ability may lead to a better understanding of the reasons for the requirement of the $\gamma$-subunit in synaptogenesis.

The ability of the C4 mutation to swap open-channel lifetime was not expected because in adult AChRs most $\alpha$-subunit mutations near the agonist sites have little or no influence on the open channel life time ${ }^{29}$. The slope of a log-log plot of opening rate versus gating equilibrium constant for a series of point mutations $(\phi)$ informs of whether the substitutions change the closed versus open lifetime, on a scale from 1 to 0 (refs 30-32). From the two sets of gating rate constants in the bimodal construct $\alpha \gamma^{\mathrm{C} 4 \delta}$ (Fig. 3b), we estimate that $\phi=0.57$, a value that is characteristic of many residues in the $\alpha$-subunit transmembrane domain ${ }^{33}$. We do not know whether this low $\phi$-value can be attributed in general to complementary-side residues or to a difference between $\gamma$ - and $\varepsilon$-subunits. An indepth exploration of amino acid $\phi$-values on the complementary surface and in the $\alpha \gamma$ core will be taken up, elsewhere.

The C4 mutation set does not account completely for differences in fetal versus adult AChR affinity. First, simulations showed a stable, high-to-low affinity exchange in $\alpha \gamma^{\mathrm{C} 4 \delta}$ but in electrophysiology experiments, the behaviour of this construct was bimodal (Fig. 3b). Apparently, with this swap, the energy barrier separating the alternative, complementary $\beta$-sheet folds was low enough to allow a reversible isomerization on the $\sim 20 \mathrm{~ms}$ time scale, which is far beyond the simulation time frame. Second, the effect of $\mathrm{C} 4$ swaps was only partial with regard to the affinity of W55A (both in silico and in vitro; Fig. 6). Third, the affinities of the $\mathrm{C} 4$ constructs for the partial agonists choline and TMA were not completely swapped (Supplementary Table 1). These results suggest that the $\mathrm{C} 4$ group does not fully account for all differences in fetal versus adult binding properties. The addition of mutations to $\mathrm{C} 4$, either from the original $\mathrm{C} 10$ set or elsewhere in the extracellular domain, might improve the fetal versus adult match in function. Also, some of the differences between simulated and experimental results may arise from longrange interactions that were not modelled.

Although the $\mathrm{C} 4$ combination was sufficient to exchange affinities, we cannot be sure that this set of substitutions is unique in this regard. There are 1,023 possible mutant combinations for the starting set of 10 , of which we examined only 84 in silico. It is possible that other combinations could also generate the affinity swap. Also, the original selection criteria could have left out important residues from the starting set. For example, $\gamma \mathrm{E} 57 /$ $\varepsilon \mathrm{G} 57 / \delta \mathrm{D} 59$ in the complementary $\beta 2$ strand were not included in the starting set because the $\varepsilon^{-}$and $\delta$-side-chains are not homologous. Nonetheless, the ability of the $\mathrm{C} 4$ combination to swap affinity and lifetime suggests that the $\mathrm{C} 4$ residues are an important basis for the functional differences between fetal versus adult endplate AChRs.

Recently, it was found that in $\alpha 4 \beta 2$ neuronal nicotinic AChRs, three complementary-subunit residues determine differential agonist potency of $\alpha 4-\alpha 4$ versus $\alpha 4-\beta 2$ binding sites ${ }^{34}$. The corresponding positions in the endplate AChR $\gamma$-subunit are L109, Y117 (in C10) and L119, none of which belong to the affinity-changing $\mathrm{C} 4$ group. This lack of correspondence may reflect a difference between these neuronal versus endplate AChRs, or may be traced to the benchmark of potency versus affinity. Nonetheless, the approach of combining simple and rapid simulations with in vitro energy estimates could be useful, in general, for revealing affinity-influencing amino acids in zones surrounding the core of ligand-binding sites in other ligand-gated ion channels.

In the simulations, the C4 mutations caused changes in structure that paralleled those that distinguished LA, $\alpha \delta^{\mathrm{WT}}$ and $\mathrm{HA}, \alpha \gamma^{\mathrm{WT}}$ sites (Fig. 8a). One of the $\mathrm{C} 4$ residues is in the $\beta 5^{\prime}$ linker, adjacent to the core, and the other three are in loop $\mathrm{E}$ and far from the core. Hence, the effects of the $\mathrm{C} 4$ side chains appear to take place by both local and non-local mechanisms, to generate a core that is more compact in HA versus LA structures (Fig. 9a).

The primary features that distinguish the complementary subunit in LA versus HA structures were as follows. (i) The $\beta 5^{\prime}$ linker is less dynamic, with the aromatic Y104 side chain close and face-on to $\alpha \mathrm{W} 149$ and suggestive of a direct, $\pi-\pi$ interaction. (ii) Loop $\mathrm{E}$ is one residue shorter. (iii) There are fewer $\mathrm{H}$-bonds near the core, at the base of the hairpin with $\mathrm{ACh}$ and near W55 in $\beta 2$. (iv) The W55 side chain is displaced from the core and not orthogonal to $\alpha \mathrm{W} 149$ or $\alpha \mathrm{Y} 93$. (v) The loop E backbone is less dynamic and the $\beta 2$ backbone near W55 is more dynamic. Overall, these differences suggest that three processes are required for $\mathrm{C} 4$ residues to generate a low affinity-unsettling the core by a local effect of the linker (104) side chain with $\alpha \mathrm{W} 149$, loosening the $\beta$-sheet near the core by reducing the probability of H-bonds, and increasing the dynamics of the W55 backbone to unlock the special pair from the core.

We hypothesize that the dynamics and orientation of 104 are both determined by the aromatic versus aliphatic character of the side chain, which in LA is Y and in HA is L. The apparent $\pi$ stacking of the $\delta$ Y104 and $\alpha \mathrm{W} 149$ aromatic rings (Fig. 9a, left) likely affects core architecture directly. This interaction may influence that between $\alpha \mathrm{W} 149$ and ACh $\left(\sim 0.7 \mathrm{kcal} \mathrm{M}^{-1}\right.$ less favourable at adult sites ${ }^{15}$ ) and could also be a reason for the lower dynamics of the $\beta 5^{\prime}$ linker. The unsettling of the core by the linker $\mathrm{Y}$ is, however, not sufficient to lower affinity completely because the effect of the point, $\mathrm{L} \leftrightarrow \mathrm{Y}$ mutation is relatively small (Fig. 5d), perhaps because $\gamma \mathrm{W} 55$ remains locked in place. Recently, we showed that the $\alpha \delta^{\mathrm{C} 4 \gamma}$ mutations reduce modal changes in affinity induced by loop C mutations at the $\alpha \delta$ site $^{35}$. It is possible that increased compactness of the site, along with the loss of a $\pi-\pi$ interaction with $\alpha \mathrm{W} 149$ stabilizes the aromatic triad and affinity.

In LA constructs the $\beta 5^{\prime}-\beta 6$ hairpin is straighter, and loop $\mathrm{E}$ is shorter and less dynamic. It is possible that these differences affect interactions between core residues and the agonist directly, but more likely they influence affinity indirectly by changing the $\mathrm{H}$-bond network of the $\beta$-sheet to increase backbone dynamics near the core. The simulations suggest that in LA structures, the bottom half of the $\beta$-hairpin and near W55, locations that have fewer H-bonds, are more dynamic (Fig. 9c). Our hypothesis is that the loop E C4 residues unlock indirectly the W55 backbone and allow this side chain to move away from the core, reducing its interaction with its partner $\alpha$ Y93 and diminishing the contribution of the special pair towards affinity.

The simulations also showed differences in the ACh molecule between LA and HA constructs. In LA structures, the ACh $\tau 2$ dihedral angle is $+60^{\circ}$ rather than $-60^{\circ}$, perhaps because the $\mathrm{H}$-bond with water is less probable as a consequence of the greater backbone dynamics nearby. However, the extent to which the differences in agonist orientation and H-bonding relate to fetal versus adult affinity is uncertain. The agonist sites show a similar affinity difference for TMA (Fig. 1c, bottom), but this ligand does not have a rotatable bond and is too distant from the water to form a $\mathrm{H}$-bond. We suspect that the core water is a factor that sets the higher affinity for ACh versus TMA, but may not be critical in determining the relative affinity of fetal versus adult sites. 
The results suggest that both the structure and dynamics of complementary $\beta$-sheet are influenced by the $\mathrm{C} 4$ amino acids as a group, and that these residues are the bases, in part, for adult versus fetal endplate receptor function. At the agonist-site core, the orientation of the 104 side chain and dynamics of the W55 backbone together appear to generate the $\sim 30$-fold affinity difference for the neurotransmitter. The simulations suggest that alternative conformations of the complementary $\beta$-sheet influence the architecture and affinity of the core. Further analyses of loop $\mathrm{E}$ interactions with loops $\mathrm{A}, \mathrm{B}$ and $\mathrm{C}$ in the $\alpha$-subunit (that hold other core aromatics) and with nearby residues in $\beta 2-\beta 3$ linker (the MIR) may further illuminate affinity mechanisms in AChRs.

\section{Methods}

Electrophysiology. AChRs were expressed in HEK cells by transient transfection of mouse endplate AChR subunits. Single-channel currents were recorded in the cell-attached patch configuration $\left(23^{\circ} \mathrm{C}\right)$. The bath solution was $(\mathrm{mM}): 142 \mathrm{KCl}$, $5.4 \mathrm{NaCl}, 1.8 \mathrm{CaCl}_{2}, 1.7 \mathrm{MgCl}_{2}, 10 \mathrm{HEPES} / \mathrm{KOH}, \mathrm{pH} 7.4$ and the pipette solution was: $137 \mathrm{NaCl}, 0.9 \mathrm{CaCl}_{2}, 2.7 \mathrm{KCl}, 1.5 \mathrm{KH}_{2} \mathrm{PO}_{4}, 0.5 \mathrm{MgCl}_{2}$ and $8.1 \mathrm{Na}_{2} \mathrm{HPO}_{4}$, $\mathrm{pH}$ 7.3. To estimate the fully liganded gating equilibrium constant, a saturating concentration of agonist $\left(100 \mathrm{mM} ;>100 \times K_{\mathrm{d}}\right)$ was added to the pipette. The agonists were acetylcholine (ACh), tetrametylammonium (TMA), carbamylcholine $(\mathrm{CCh})$ and choline. To place the gating rate constants into a range suitable for kinetic analysis, we added background mutations that changed the allosteric constant or changed the membrane voltage. Neither of these perturbations had any effect on affinity ${ }^{36,37}$.

Experimental estimates of affinity and binding energy. Resting-state binding energy (in $\mathrm{kcal} \mathrm{M}^{-1}$ ) is equal to $+0.59 \ln K_{\mathrm{d}}\left(23^{\circ} \mathrm{C}\right)$. $K_{\mathrm{d}}$ was estimated from single-channel current interval durations by using QUB software ${ }^{38}$. In one approach (Fig. $3 \mathrm{a}$ ), $K_{\mathrm{d}}$ was estimated from the ratio of dissociation/association rate constants, which in turn were estimated by fitting interval durations across multiple [agonist] using a $\mathrm{A}+\mathrm{C} \leftrightarrow \mathrm{AC} \leftrightarrow \mathrm{AO}$ scheme (one-site AChRs; $\mathrm{A}$ is the agonist, $\mathrm{C}$ is closed and $\mathrm{O}$ is open). In another approach (Figs 4 and $5 \mathrm{~d}$; Supplementary Table 1 ), $K_{\mathrm{d}}$ was estimated from a ratio of gating equilibrium constants, as follows. In both WT and mutant AChRs, $K_{\mathrm{d}} \approx E_{0} / E_{1}$, where $E$ is the $\mathrm{C} \leftrightarrow \mathrm{O}$ gating equilibrium constant and the subscript indicates the number of bounds agonist $\left(E_{0} \text { is the allosteric constant }\right)^{39} . E_{1}$ was estimated as the gating equilibrium constant for a single site at high (agonist; full saturation, $>5 \times K_{\mathrm{d}}$ ). $E_{0}$ was estimated from the gating equilibrium constant in the absence of any agonists using a constitutively active background ${ }^{40,41}$. In the cross-concentration approach, the membrane was held at $-100 \mathrm{mV}$, and in experiments at high (agonist) the membrane was depolarized to $+70 \mathrm{mV}$ to reduce channel block by the agonist. Membrane potential has no effect on $K_{\mathrm{d}}$ (ref. 37). When both approaches were used for the same construct, the $K_{\mathrm{d}}$ estimates agreed.

Protein engineering. The mutations were incorporated into AChR subunits using the QuickChange site-directed mutagenesis kit (Agilent Technologies, CA) and were verified by nucleotide sequencing. Many AChR mutations away from the agonist sites only influence $E_{0}$ (ref. 33), which is 7.4 or $0.52 \times 10^{-7}$ in WT adult or fetal AChRs at $-100 \mathrm{mV}$ (refs 40,41 ). We measured $E_{0}$ for every mutant construct by adding background mutations that increased it by known extents, but had no effect on affinity ${ }^{36}$. In selecting the backgrounds, we chose those that were energetically independent, so that the aggregate $E_{0}$ was the product of the WT value and the individual fold changes. The values shown in Fig. $5 \mathrm{~d}$ have been corrected for the backgrounds.

To study AChRs having just one functional binding site, we added mutations to the $\varepsilon^{-}, \gamma-, \delta$-subunits that eliminate binding at the mutated site ${ }^{24}$. To make $\alpha \gamma$ - or $\alpha \varepsilon$-only AChRs, we added $\delta \mathrm{P} 123 \mathrm{R}$, and to make $\alpha \delta$-only, we added $\varepsilon / \gamma \mathrm{P} 121 \mathrm{R}$, sometimes in combination with $\gamma \mathrm{W} 55 \mathrm{R}$. These mutations also change $E_{0}$, which was measured for each knock-out construct.

Homology model. The homology model of the extracellular domain of the fetaltype AChR was built based on the Aplysia californica ACh binding protein (AChBP) bound to epibatidine (pdb ID: 2BYQ) by using MODELLER ${ }^{42}$. The sequences of AChBP and AChR subunits were aligned using CLUSTALX ${ }^{43}$ (Fig. 10a). The AChR subunits share $\sim 20 \%$ sequence identity with Aplysia AChBP. AChR subunits were modelled simultaneously so that spatial reciprocity was maintained at the interfaces. Residues 128 and 142 in the $\alpha$-subunit and the corresponding residues in the other subunits were constrained as disulfide bonds. A protocol of conjugate gradient optimization, simulated annealing and molecular dynamics were used to refine the structure.

First, 100 structural models were generated. MODELLER has various assessment methods and objective functions to test the validity of a homology model (such as molpdf and DOPE scores), but these are not recommended to be used for multi-chain proteins ${ }^{44}$. Therefore, we used PROCHECK scores based on G-factor ${ }^{45}$ to rank the models. The model with the best G-factor score was chosen for docking and simulations ${ }^{46}$. The top five models based on G-factor were similar in both structure (backbone root-mean-square deviation (RMSD) $<0.7 \AA$; the equilibrated structure RMSD from the simulations was $1.2 \AA$ ) and scores. The selected model also ranked high in the MODELLER scores (first in molpdf and fifth in DOPE score), bad contacts (third) and Ramachandran criteria (third). Further minimization of the selected model reduced the bad contacts to zero.

Ligand docking. ACh and TMA were docked at the $\alpha \delta$ and $\alpha \gamma$ binding sites using the Lamarckian genetic algorithm in AUTODOCK ${ }^{47}$. ACh and the core aromatic residues were allowed to be flexible, and $30 \AA$ cubic search grid was used at the expected binding site with $0.375 \AA$ grid spacing. Docked structures were analysed and selected on the basis of lowest energy and RMSD clustering. The CHARMM force field parameters for ACh and TMA were obtained from the Charmm Generalized Small Molecule Force Field webserver (CGenFF) ${ }^{48,49}$.

MD simulations. Point mutations were introduced in silico using the VMD mutator plug-in ${ }^{50}$. AChRs with ligands or mutations were optimized and equilibrated by using energy minimization and MD simulation. The system was solvated in a water box with TIP3P water model ${ }^{51}$ and the box boundary was extended at least to $10 \AA$ from the periphery of the protein in each dimension. $\mathrm{Na}^{+}$ and $\mathrm{Cl}^{-}$ions were added to neutralize the system and bring it to an ionic concentration of $150 \mathrm{mM} \mathrm{NaCl}$.

Molecular dynamics simulations were run using NAMD ${ }^{52}$ version 2.8 , with the CHARMM27 force field ${ }^{53}$. First, a 20,000-step minimization was done using the steepest descent method, and with gradual release of restraints on the protein backbone. The system was heated to $300 \mathrm{~K}$ over 100 ps, a 500 ps equilibration run was performed in the NVT ensemble and then $20 \mathrm{~ns}$ MD simulations were performed in the NPT ensemble at a temperature of $300 \mathrm{~K}$ and pressure of $1 \mathrm{~atm}$ using the Nosé-Hoover method ${ }^{54}$. Following minimization, harmonic constraints (force constant $=1 \mathrm{kcal} \mathrm{M}^{-1} \AA^{-2}$ ) were applied on the $\mathrm{C} \alpha$ atoms of residues, which were $>25 \AA$ away from the ligand. These restraints maintained the global backbone conformation of the model while allowing relaxation of all side chains.

Periodic boundary conditions were applied. A $10 \AA$ switching distance and a $12 \AA$ cutoff distance were used for non-bonded interactions. The particle mesh Ewald method ${ }^{55}$ was used to calculate long-range electrostatic interactions. The SHAKE algorithm ${ }^{56}$ was used to constrain bond lengths of hydrogen-containing bonds, which allows a time step of 2 fs for MD simulations. Four MD simulation trajectories were obtained for each system. The coordinates of the systems were saved every 1 ps during MD simulations for later analyses. The protein RMSD became stable by the first $10 \mathrm{~ns}$ (Fig. 10b). All the analyses and binding energy calculations were done on snapshots extracted every 20 ps over the last $10 \mathrm{~ns}$ of each trajectory. The ensemble for each system therefore contained 2,000 snapshots of the system.

Calculation of affinity. A total ligand-protein binding energy $(\Delta E)$ was estimated as described elsewhere ${ }^{57}$. In brief, this energy was calculated using a continuum solvent model from an ensemble of 2,000 snapshots:

$$
\Delta E=\alpha E_{\mathrm{vdW}}+\beta \Delta E_{\text {elec }}
$$

where $E_{\mathrm{vdW}}$ is the van der Waals contribution and $\Delta E_{\text {elec }}$ and is the electrostatic contribution calculated using the Poisson - Boltzmann (PB) method (PBEQ module of CHARMM ${ }^{58}$ in a salt concentration of $140 \mathrm{mM}$ ). The molecular dielectric surface was defined by a probe radius of $1.4 \AA$. Previously optimized atomic Born radii ${ }^{59}$ for the 20 amino acids were used to estimate the electrostatic free energy in explicit water molecules. The dielectric constant of the protein interior and the aqueous environment were set to 4 and 80 , respectively.

The coefficients $\alpha$ and $\beta$ in equation (1) were evaluated by comparing simulated and experimental $\Delta E$ values for alanine mutations and optimizing the coefficients to reproduce the experimental energies (Fig. 1b). We calculated in silico binding energies for the WT (with ACh/TMA) and Ala mutations at each of the core aromatic residues $(\alpha 93, \alpha 149, \alpha 190, \alpha 198$ and $\delta 55)$, as described above. Values of $\alpha$ and $\beta$ were scanned within a range of $0-2$ to minimize the RMSE (root-mean-square error) between simulated and experimental energies for total 13 sample points:

$$
\operatorname{RMSE}=\left[\frac{1}{N} \sum_{n}\left(\Delta E_{n}-\Delta E_{\exp , n}\right)^{2}\right]^{1 / 2}
$$

where $n$ is each sample point and $N=13$. The overall fitting quality was tested by the coefficient of multiple determination $R^{2}$. The result was $\alpha=0.22$ and $\beta=1.24$. The $\alpha$ and $\beta$ values for barnase-barstar ( 0.17 and 1 (ref. 57)) were shown previously to reproduce the fetal versus adult ACh binding energy difference ${ }^{15}$. The small $\alpha$ is possibly due to the loss of protein-water VdW contacts in the AChR, whereas the larger $\beta(>1)$ may be due to cation $-\pi$ interactions between the quaternary ammonium group of the ligand and the binding site aromatics, which is unaccounted for by the classical force field. With these values of $\alpha$ and $\beta$, the RMSE between simulated and experimental results were at a minimum $\left(0.34 \mathrm{kcal} \mathrm{M}^{-1} ; R^{2}=0.92\right)$, with a correlation coefficient of 0.97 . To test the generality of these parameters, we 
a

AChR alpha1
AChR epsilon
AChR gamma
AChR delta
AChBP Aplysia
AChBP Lymnaea
AChR alpha1
AChR epsilon
AChR gamma
AChR delta
AChBP Aplysia
AChBP Lymnaea

AChR alpha1
AChR epsilon
AChR gamma
AChR delta
AChBP Aplysia
AChBP Lymnaea
AChR alpha1
AChR epsilon
AChR gamma
AChR delta
AChBP Aplysia
AChBP Lymnaea

17 SVVRPVEDHREIVQVTVGLQLIQLINVDEVNQIVTTINVRLKQQWVDYNLKWNPDDYGGVK 76 17 PECRPVRRPEDTVTITLKVTLTNLISLNEKEETLTTSVWIGIDWHDYRLNYSKDDFAGVG 76 17 PHLRPAERDSDVVNVSLKLTLTNLISLNEREEALTTNVWIEMQWCDYRLRWDPKDYEGLW 76 19 KDLRPVARKEDKVDVALSLTLSNLISLKEVEETLTTNVWIDHAWVDSRLQWDANDFGNIT 78 17 SPMYPGPTKDDPLTVTLGFTLQDIVKADSSTNEVDLVYYEQQRWKLNSLMWDPNEYGNIT 76 15 RPDVIPTQRDRPVAVSVSLKFINILEVNEITNEVDVVFWOQTTWSDRTLAWNSSHSP - - D 72

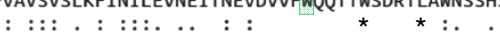

77 KIHIPSEKIWRPDVVLYNNADGDFAIVKFTKVLLDYTGHITWTPPAIFKSYCEIIVTHFP 136 77 IIRVPSEHVW 77 ITROPTMVR 77 ILRVPSTMVWRPIVLENMVCVEEVALYCNULVSPOCCIYWLPPAIFRSSCSISVTYFP 136 77 VLRLPPOMUWLPEIVLENMNDSPQISYACNULVYDSCIVIWLPPAIFRSSCPISVTYFP 138 77 DFRTSAADIWTPDITAYSSTR - PVQVLSPQIAVVTHDGSVMFIPAQRLSFMCDPTGVD-S 134 73 QVSVPISSLWVPDLAAYNAIS-KPEVLTPQLARVVSDGEVLYMPSIRQRFSCDVSGVD-T 130 $: * \star::$.

137 FDEQNCSMKLGTWTYDGSVVAINPESDQP. . . . . . . . . . . . . DLSNFMESGEWVIKEARGW 184 137 FDWQNCSLIFRSOTYNAEEVEFIFAVDDD - - - GNTINKIDIDTAAFTENGEWAIDYCPGM 193 137 FDWQNCSLIFQSPTYSTSEINLQLSQEDG -..-PAIEWIFIDPEAFTENGEWAIRHRPAK 192 139 FDWQNCSLKFSSLKYTAKEITLSLKQEEENMRSYPIEWIIIDPEGFTENGEWEIVHRAAK 198 (3) 131 EEATCAVKFGSWYSCEETDLKTDTDQV-

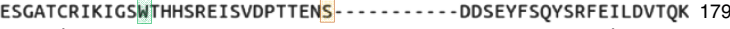

$$
\text { .* : : : : : : : : } \quad \text { : . }
$$

85 KHWVFYSCCPT - . - - TPYLDITYHFVMQRLP 211 194 IR - -RYEGGST - - EGPGETDVIYTLIIRRKP 220 93 ML - - LDSVAPA - -EEAGHQKVVFYLLIQRKP 219 199 LN - -VDPSVPM --DSTNHODVTFYLIIRRKP 225 83 RQVQHYSCCPE.......PYIDVNLVVKFRERR 208 180 KNSVTYSCCPE........AYEDVEVSLNFRKKG 205 $\therefore \quad .::$.

b Backbone RMSD $(\AA ̊)$

C

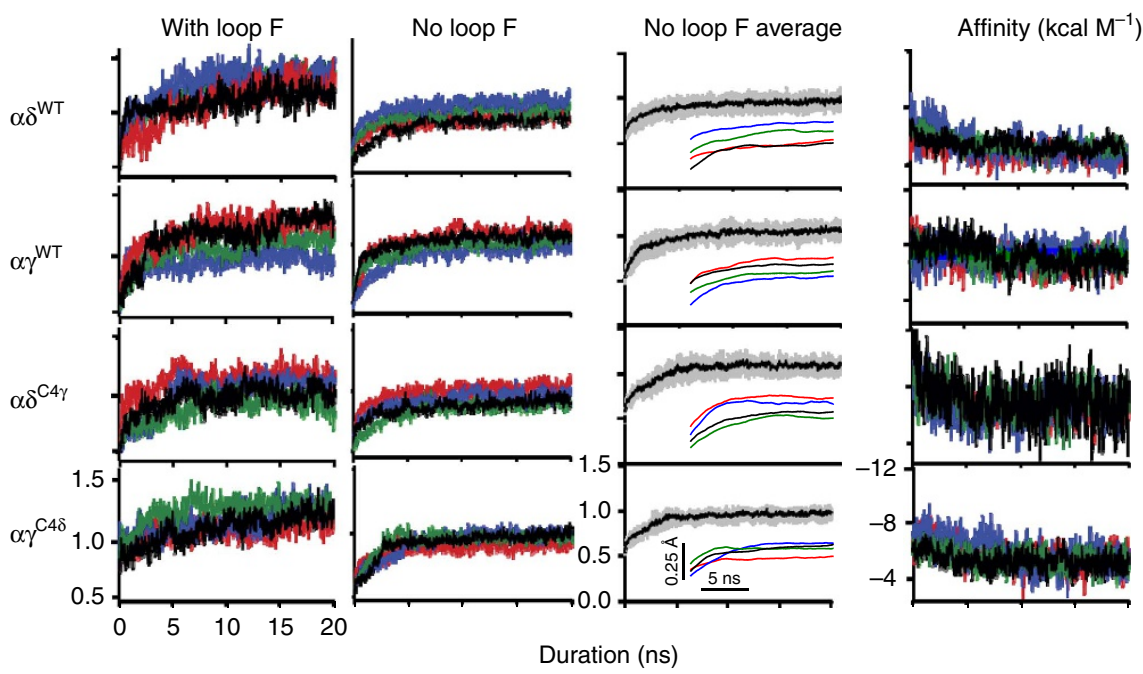

Figure 10 | Sequence alignment and stability of models. (a) Sequence alignment of the extracellular domain of AChR subunits (mouse) and AChBPs. Highlighted residues are: core aromatics, green; C4, violet; other members of C10, orange. (b) Temporal evolution of simulated root-mean-square-deviation (RMSD) in WT and C4-swapped constructs. Left, total RMSD; each colour is a different trajectory. Centre, RMSD after removing a section of loop F. Right, average (black) + s.d. (grey) of all four trajectories (loop F removed; inset is rolling $5 \mathrm{~ns}$ average of each trajectory). All three plots show that the structure is stabilized by $\sim 10 \mathrm{~ns}$. (c) Temporal evolution of simulated ACh binding energy (loop F included).

plotted simulated and experimental affinities for two additional, non-alanine mutations ( $\delta \mathrm{Y} 106 \mathrm{~L}$ and $\gamma \mathrm{L} 104 \mathrm{Y})$ in Fig. 1b. These fell on the same regression line.

The approximate binding energy calculated from MD simulations using the above method $(\Delta E)$ and the free energy estimated from single-channel currents $\left(+0.59 \ln \left(K_{\mathrm{d}}\right)\right)$ are not equivalent. However, the entropy contribution to ligand binding energy has been experimentally shown to be negligible in case of muscletype $\mathrm{AChRs}^{22}$, and here we show that changes in these quantities can be compared for the purpose of engineering biological AChRs. In the text, we use 'affinity' for both the in silico and in vitro binding energy estimates.

Structure analyses. The geometric centres of the aromatic rings of interest and the ACh quaternary amine (QA) nitrogen were used as reference points. Structural analyses were done using the last $10 \mathrm{~ns}$ of each trajectory for the hetro-pentamer simulations. We measured all the distances and angles using VMD.

The volume of the ligand binding pocket was calculated by joining the centroids of the aromatic rings to form two adjoining tetrahedrons. The volume of each tetrahedron was calculated using the three-simplex determinant method from the coordinates of the vertices, as described elsewhere ${ }^{15}$. The $\beta$-hairpin twist was measured from the angle between the central axial vectors of $\beta 5^{\prime}$ and $\beta 6$. The central axis was defined by the least squares linear regression fit of the coordinates of the backbone atoms of residues $107-110$ in $\beta 5^{\prime}$ and residues $115-118$ in $\beta 6$
( $\gamma$-subunit numbers). The regression fit was calculated by singular value decomposition of the coordinates.

To identify a hydrogen bond between two atoms (that is, acceptor and donor) a donor-acceptor distance of $<3.5 \AA$ and a H-donor-acceptor angle of $\leq 30^{\circ}$ were used as criteria ${ }^{60}$. We used VMD to identify and calculate the occupancies of all H-bonds within the last 10-ns ensemble.

RMSD and RMSF analyses. To assess the conformational stability of the MD simulations, we calculated the RMSD of all backbone atoms in the $\alpha$-plus complementary subunit relative to the starting structures. The RMSD plots showed a small amount of drift over time in some of the trajectories (Fig. 10b, left). We determined that most of this drift came from the complementary-side loop F, which was highly flexible and disorganized. We repeated the RMSD analyses after removing a part of loop F from the calculation (residues 166-183 in $\gamma$ and 164-177 in $\delta$ ), and both the drift and variance in the RMSD were reduced significantly (Fig. 10b, centre). The plots show that the system stabilized within $\sim 10 \mathrm{~ns}$. This region of loop $\mathrm{F}$ had been modelled ab-initio into the homology model because there is no corresponding region in AChBP. To test the role of loop F in affinity, we partitioned the binding energy (Equation 1) into contributions from individual residues using CHARMM. None of the loop F residues that were excluded from the RMSD calculations were in the top $5 \%(n \sim 25)$ of those contributing to affinity, 
that comprise $\sim 98 \%$ of the total binding energy. The choice of $5 \%$ corresponds to a $P$ value of 0.05 .

As a further test of stability, we calculated a running average on the RMSD (without loop F) using a rolling window of $5 \mathrm{~ns}$ (Fig. 10b, right inset). The slopes of the regression lines fitted to the last $10 \mathrm{~ns}$ were small. The overall mean absolute slope value was $0.002+0.001 \AA^{n n^{-1}}$ ( + s.d) for all the four constructs combined ( $n=16$ trajectories). In addition, we calculated the average RMSD for the four trajectories for each construct (Fig. 10b, right; s.d. calculated from 5-ns bins). The drifts in these averages during the last $10 \mathrm{~ns}(\sim 0.02 \AA)$ were smaller than the s.d.s. of the fluctuations $(\sim 0.07 \AA)$. These tests establish that all of the systems became stable within $10 \mathrm{~ns}$ and could be used for affinity estimation.

To compare the flexibility of ligand binding interface between the two states, we performed RMSF analysis on the backbone atoms based on the last $10 \mathrm{~ns}$ of the MD simulations. The RMSF of each residue was calculated with respect to the average structure of the ensemble using VMD version-1.9. This gives a residue-wise average of all possible fluctuations in the trajectories.

\section{References}

1. Nayak, T. K. \& Auerbach, A. Asymmetric transmitter binding sites of fetal muscle acetylcholine receptors shape their synaptic response. Proc. Natl Acad. Sci. USA 110, 13654-13659 (2013).

2. Jackson, M. B. Dependence of acetylcholine receptor channel kinetics on agonist concentration in cultured mouse muscle fibres. J. Physiol. 397, 555-583 (1988).

3. Salamone, F. N., Zhou, M. \& Auerbach, A. A re-examination of adult mouse nicotinic acetylcholine receptor channel activation kinetics. J. Physiol. 516, 315-330 (1999).

4. Sine, S. M., Claudio, T. \& Sigworth, F. J. Activation of Torpedo acetylcholine receptors expressed in mouse fibroblasts. Single channel current kinetics reveal distinct agonist binding affinities. J. Gen. Physiol. 96, 395-437 (1990).

5. Brejc, K. et al. Crystal structure of an ACh-binding protein reveals the ligandbinding domain of nicotinic receptors. Nature 411, 269-276 (2001).

6. Jaramillo, F., Vicini, S. \& Schuetze, S. M. Embryonic acetylcholine receptors guarantee spontaneous contractions in rat developing muscle. Nature 335, 66-68 (1988).

7. Witzemann, V. et al. Acetylcholine receptor epsilon-subunit deletion causes muscle weakness and atrophy in juvenile and adult mice. Proc. Natl Acad. Sci. USA 93, 13286-13291 (1996)

8. Liu, Y. et al. Essential roles of the acetylcholine receptor gamma-subunit in neuromuscular synaptic patterning. Development 135, 1957-1967 (2008).

9. Takahashi, M. et al. Spontaneous muscle action potentials fail to develop without fetal-type acetylcholine receptors. EMBO Rep. 3, 674-681 (2002).

10. Hoffmann, K. et al. Escobar syndrome is a prenatal myasthenia caused by disruption of the acetylcholine receptor fetal gamma subunit. Am. J. Hum. Genet. 79, 303-312 (2006)

11. Morgan, N. V. et al. Mutations in the embryonal subunit of the acetylcholine receptor (CHRNG) cause lethal and Escobar variants of multiple pterygium syndrome. Am. J. Hum. Genet. 79, 390-395 (2006).

12. Vogt, J. et al. CHRNG genotype-phenotype correlations in the multiple pterygium syndromes. J. Med. Genet. 49, 21-26 (2012).

13. Mishina, M. et al. Molecular distinction between fetal and adult forms of muscle acetylcholine receptor. Nature 321, 406-411 (1986).

14. Villarroel, A. \& Sakmann, B. Calcium permeability increase of endplate channels in rat muscle during postnatal development. J. Gen. Physiol. 496, 331-338 (1996).

15. Nayak, T. K. et al. Functional differences between neurotransmitter binding sites of muscle acetylcholine receptors. Proc. Natl Acad. Sci. USA 111, 17660-17665 (2014).

16. Polak, R. L., Sellin, L. C. \& Thesleff, S. Acetylcholine content and release in denervated or botulinum poisoned rat skeletal muscle. J. Physiol. 319, 253-259 (1981).

17. Bouzat, C., Bren, N. \& Sine, S. M. Structural basis of the different gating kinetics of fetal and adult acetylcholine receptors. Neuron 13, 1395-1402 (1994).

18. Sullivan, M. P., Owens, J. L. \& Kullberg, R. W. Role of M2 domain residues in conductance and gating of acetylcholine receptors in developing Xenopus muscle. J. Physiol. 515, 31-39 (1999).

19. Herlitze, S., Villarroel, A., Witzemann, V., Koenen, M. \& Sakmann, B. Structural determinants of channel conductance in fetal and adult rat muscle acetylcholine receptors. J. Physiol. 492, 775-787 (1996).

20. Auerbach, A. Agonist activation of a nicotinic acetylcholine receptor. Neuropharmacology 96, 150-156 (2015).

21. Purohit, P., Bruhova, I. \& Auerbach, A. Sources of energy for gating by neurotransmitters in acetylcholine receptor channels. Proc. Natl Acad. Sci. USA 109, 9384-9389 (2012).

22. Gupta, S. \& Auerbach, A. Temperature dependence of acetylcholine receptor channels activated by different agonists. Biophys. J. 100, 895-903 (2011).

23. Celie, P. H. et al. Nicotine and carbamylcholine binding to nicotinic acetylcholine receptors as studied in AChBP crystal structures. Neuron 41, 907-914 (2004)
24. Gupta, S., Purohit, P. \& Auerbach, A. Function of interfacial prolines at the transmitter binding sites of the neuromuscular acetylcholine receptor. J. Biol. Chem. 288, 12667-12679 (2013).

25. Bafna, P. A., Jha, A. \& Auerbach, A. Aromatic residues \{epsilon\}Trp-55 and \{delta\}Trp-57 and the activation of acetylcholine receptor channels. J. Biol. Chem. 284, 8582-8588 (2009).

26. Xie, Y. \& Cohen, J. B. Contributions of Torpedo nicotinic acetylcholine receptor gamma Trp-55 and delta Trp-57 to agonist and competitive antagonist function. J. Biol. Chem. 276, 2417-2426 (2001).

27. Olsen, J. A., Balle, T., Gajhede, M., Ahring, P. K. \& Kastrup, J. S. Molecular recognition of the neurotransmitter acetylcholine by an acetylcholine binding protein reveals determinants of binding to nicotinic acetylcholine receptors. PLoS ONE 9, e91232 (2014).

28. Amiri, S., Sansom, M. S. \& Biggin, P. C. Molecular dynamics studies of AChBP with nicotine and carbamylcholine: the role of water in the binding pocket. Protein Eng. Des. Sel. 20, 353-359 (2007).

29. Grosman, C., Zhou, M. \& Auerbach, A. Mapping the conformational wave of acetylcholine receptor channel gating. Nature 403, 773-776 (2000).

30. Fersht, A. R., Leatherbarrow, R. J. \& Wells, T. N. C. Quantitative analysis of structure-activity relationships in engineered proteins by linear free-energy relationships. Nature 322, 284-286 (1986).

31. Fersht, A. R., Leatherbarrow, R. J. \& Wells, T. N. Structure-activity relationships in engineered proteins: analysis of use of binding energy by linear free energy relationships. Biochemistry 26, 6030-6038 (1987).

32. Estell, D. A. et al. Probing steric and hydrophobic effects on enzyme-substrate interactions by protein engineering. Science 233, 659-663 (1986).

33. Purohit, P., Gupta, S., Jadey, S. \& Auerbach, A. Functional anatomy of an allosteric protein. Nat. Commun. 4, 2984 (2013).

34. Shahsavar, A. et al. AChBP engineered to mimic the alpha4-beta4 binding pocket in alpha4beta2 nicotinic acetylcholine receptors reveals interface specific interactions important for binding and activity. Mol. Pharmacol. 88, 697-707 (2015).

35. Vij, R., Purohit, P. \& Auerbach, A. Modal affinities of endplate acetylcholine receptors caused by loop C mutations. J. Gen. Physiol. 146, 375-386 (2015).

36. Jadey, S. V., Purohit, P., Bruhova, I., Gregg, T. M. \& Auerbach, A. Design and control of acetylcholine receptor conformational change. Proc. Natl Acad. Sci. USA 108, 4328-4333 (2011).

37. Auerbach, A., Sigurdson, W., Chen, J. \& Akk, G. Voltage dependence of mouse acetylcholine receptor gating: different charge movements in di-, mono- and unliganded receptors. J. Physiol. 494, 155-170 (1996).

38. Nicolai, C. \& Sachs, F. Solving ion channel kinetics with the QuB software. Biophys. Rev. Lett. 08, 1-21 (2013).

39. Purohit, P., Bruhova, I., Gupta, S. \& Auerbach, A. Catch-and-hold activation of muscle acetylcholine receptors having transmitter binding site mutations. Biophys. J. 107, 88-99 (2014).

40. Purohit, P. \& Auerbach, A. Unliganded gating of acetylcholine receptor channels. Proc. Natl Acad. Sci. USA 106, 115-120 (2009).

41. Nayak, T. K., Purohit, P. G. \& Auerbach, A. The intrinsic energy of the gating isomerization of a neuromuscular acetylcholine receptor channel. J. Gen. Physiol. 139, 349-358 (2012).

42. Eswar, N., Eramian, D., Webb, B., Shen, M. Y. \& Sali, A. Protein structure modeling with MODELLER. Methods Mol. Biol. 426, 145-159 (2008).

43. Hansen, S. B. et al. Structures of Aplysia AChBP complexes with nicotinic agonists and antagonists reveal distinctive binding interfaces and conformations. EMBO J. 24, 3635-3646 (2005).

44. Shen, M. Y. \& Sali, A. Statistical potential for assessment and prediction of protein structures. Protein Sci. 15, 2507-2524 (2006).

45. Engh, R. A. \& Huber, R. Accurate bond and angle parameters for X-ray protein-structure refinement. Acta Crystallogr. A 47, 392-400 (1991).

46. Laskowski, R. A., MacArthur, M. W., Moss, D. S. \& Thornton, J. M. PROCHECK - a program to check the stereochemical quality of protein structures. J. Appl. Crystallogr. 26, 283-291 (1993).

47. Morris, G. M. et al. Autodock4 and AutoDockTools4: automated docking with selective receptor flexiblity. J. Comp. Chem. 16, 2785-2791 (2009).

48. Vanommeslaeghe, K. \& MacKerell, Jr A. D. Automation of the CHARMM General Force Field (CGenFF) I: bond perception and atom typing. J. Chem. Inf. Model. 52, 3144-3154 (2012).

49. Vanommeslaeghe, K., Raman, E. P. \& MacKerell, Jr A. D. Automation of the CHARMM General Force Field (CGenFF) II: assignment of bonded parameters and partial atomic charges. J. Chem. Inf. Model. 52, 3155-3168 (2012).

50. Humphrey, W., Dalke, A. \& Schulten, K. VMD: visual molecular dynamics. J. Mol. Graph. 14, 33-38 (1996).

51. Foloppe, N. \& Mackerell, A. D. All-atom empirical force field for nucleic acids: I. Parameter optimization based on small molecule and condensed phase macromolecular target data. J. Comp. Chem. 21, 86-104 (2000).

52. Phillips, J. C. et al. Scalable molecular dynamics with NAMD. J. Comp. Chem. 26, 1781-1802 (2005). 
53. MacKerell, A. D. et al. All-atom empirical potential for molecular modeling and dynamics studies of proteins. J Phys. Chem. B 102, 3586-3616 (1998).

54. Martyna, G. J., Hughes, A. \& Tuckerman, M. E. Molecular dynamics algorithms for path integrals in constant pressure. J. Chem. Phys. 110, 3275-3290 (1999).

55. Deserno, M. \& Holm, C. How to mesh up Ewald sums. I. A theoretical and numerical comparison of various particle mesh routines. J. Chem. Phys. 109, 7678-7693 (1998).

56. Hoover, W. G. Canonical dynamics: equilibrium phase-space distributions. Phys. Rev. A 31, 1695-1697 (1985).

57. Eriksson, M. A. \& Roux, B. Modeling the structure of agitoxin in complex with the Shaker $\mathrm{K}+$ channel: a computational approach based on experimental distance restraints extracted from thermodynamic mutant cycles. Biophys. J. 83, 2595-2609 (2002).

58. Brooks, B. R. et al. A program for macromolecular energy, minimization, and dynamics calculations. J. Comp. Chem. 4, 187-217 (1983).

59. Nina, M., Beglov, D. \& Roux, B. Atomic radii for continuum electrostatics calculations based on molecular dynamics free energy simulations. J. Phys. Chem. B 101, 5239-5248 (1997).

60. Ashby, J. A. et al. GABA binding to an insect GABA receptor: a molecular dynamics and mutagenesis study. Biophys. J. 103, 2071-2081 (2012).

\section{Acknowledgements}

We thank M. Merritt, M. Shero and M. Teeling for technical assistance and the CCR, UB for computational facilities. This work was funded by NIH (NS064969).

\section{Author contributions}

T.K.N., S.C. and A.A. conceptualized the project; T.K.N., S.C., W.Z. and A.A. designed the experiments; T.K.N. performed the electrophysiology; S.C. performed the simulations; T.K.N., S.C. and A.A. analysed the data; T.K.N., S.C., W.Z. and A.A. interpreted the data; A.A. prepared the manuscript.

\section{Additional information}

Supplementary Information accompanies this paper at http://www.nature.com/ naturecommunications

Competing financial interests: The authors declare no competing financial interests.

Reprints and permission information is available online at http://npg.nature.com/ reprintsandpermissions/

How to cite this article: Nayak, T. K. et al. Structural correlates of affinity in fetal versus adult endplate nicotinic receptors. Nat. Commun. 7:11352 doi: 10.1038/ncomms11352 (2016)

(c) This work is licensed under a Creative Commons Attribution 4.0 International License. The images or other third party material in this article are included in the article's Creative Commons license, unless indicated otherwise in the credit line; if the material is not included under the Creative Commons license, users will need to obtain permission from the license holder to reproduce the material. To view a copy of this license, visit http://creativecommons.org/licenses/by/4.0/ 